\title{
Transcription Factors in Alkaloid Engineering
}

\author{
Yasuyuki Yamada $1, *$ (D) and Fumihiko Sato $2,3, *$ (D) \\ 1 Laboratory of Medicinal Cell Biology, Kobe Pharmaceutical University, Kobe 658-8558, Japan \\ 2 Department of Plant Gene and Totipotency, Division of Integrated Life Science, Graduate School of Biostudies, \\ Kyoto University, Kyoto 606-8502, Japan \\ 3 Graduate School of Science, Osaka Prefecture University, Sakai 599-8531, Japan \\ * Correspondence: yyamada@kobepharma-u.ac.jp (Y.Y.); fsato@lif.kyoto-u.ac.jp (F.S.); \\ Tel.: +81-78-441-7544 (Y.Y.)
}

\begin{abstract}
Plants produce a large variety of low-molecular-weight and specialized secondary compounds. Among them, nitrogen-containing alkaloids are the most biologically active and are often used in the pharmaceutical industry. Although alkaloid chemistry has been intensively investigated, characterization of alkaloid biosynthesis, including biosynthetic enzyme genes and their regulation, especially the transcription factors involved, has been relatively delayed, since only a limited number of plant species produce these specific types of alkaloids in a tissue/cell-specific or developmentalspecific manner. Recent advances in molecular biology technologies, such as RNA sequencing, co-expression analysis of transcripts and metabolites, and functional characterization of genes using recombinant technology and cutting-edge technology for metabolite identification, have enabled a more detailed characterization of alkaloid pathways. Thus, transcriptional regulation of alkaloid biosynthesis by transcription factors, such as basic helix-loop-helix (bHLH), APETALA2/ethyleneresponsive factor (AP2/ERF), and WRKY, is well elucidated. In addition, jasmonate signaling, an important cue in alkaloid biosynthesis, and its cascade, interaction of transcription factors, and post-transcriptional regulation are also characterized and show cell/tissue-specific or developmental regulation. Furthermore, current sequencing technology provides more information on the genome structure of alkaloid-producing plants with large and complex genomes, for genome-wide characterization. Based on the latest information, we discuss the application of transcription factors in alkaloid engineering.
\end{abstract}

Academic Editors:

Kyoungwhan Back,

Roque Bru-Martinez,

Giulia Malacarne and José

Tomás Matus

Received: 14 October 2021

Accepted: 16 November 2021

Published: 18 November 2021

Publisher's Note: MDPI stays neutral with regard to jurisdictional claims in published maps and institutional affiliations.

Keywords: benzylisoquinoline alkaloid; monoterpene indole alkaloid; nicotine; steroidal glycoalkaloid; transcription factor; jasmonate signaling; gene cluster; metabolic engineering; transcriptional network

\section{Introduction}

Plants produce structurally divergent low-molecular-weight secondary metabolites (recently termed as "specialized metabolites") to protect their bodies from the attack of pathogens and herbivores, and/or to interact with beneficial organisms, such as to attract symbiotic microorganisms and pollinators. Among secondary metabolites, nitrogencontaining alkaloids found in a limited number of plant species are often more biologically active and are thus used as pharmaceuticals [1]. Because of the diversity of the chemical structures and biosynthetic/evolutional origins of such compounds, the elucidation of their biosynthetic pathways and regulatory mechanisms of biosynthesis has been limited to a few plant species. In this review, we focus on transcription factors (TFs) that regulate alkaloid biosynthesis in comparison with those of more universal secondary metabolites, such as phenylpropanoids and terpenoids and their potential use in alkaloid engineering.

\section{General Characteristics of TFs}

TFs regulate gene expression involved in numerous plant processes through specifically binding to cis-acting elements in the promoters of target genes. Several TFs involved 
in the regulation of alkaloid biosynthesis have been characterized, including those of nicotine alkaloids in Nicotiana species; steroidal glycoalkaloids (SGAs) such as tomatine in Solanum lycopersicum (Solanaceae) and solanine in Solanum tuberosum (Solanaceae); monoterpenoid indole alkaloids (MIAs) such as vinblastine and vincristine in Catharanthus roseus (Apocynaceae); and benzylisoquinoline alkaloids (BIAs) such as berberine in Coptis japonica (Ranunculaceae), sanguinarine in Eschscholzia californica (Papaveraceae), and morphine in Papaver somniferum (Papaveraceae) [2,3]. Detailed TFs reported in the alkaloid pathways are listed in Table 1 and described below.

Table 1. TFs that have been identified in alkaloid biosynthesis.

\begin{tabular}{|c|c|c|c|c|}
\hline Family & Name & Accession No. or Gene ID & Plant Species & Reference \\
\hline \multirow{16}{*}{$\mathrm{AP} 2 / \mathrm{ERF}$} & ORCA2 & AJ238740 & Catharanthus roseus & {$[4]$} \\
\hline & ORCA3 & EU072424 & Catharanthus roseus & [5] \\
\hline & ORCA4 & KR703577 & Catharanthus roseus & [6] \\
\hline & ORCA5 & KR703578 & Catharanthus roseus & [7] \\
\hline & ORCA6 & MN614455 & Catharanthus roseus & [8] \\
\hline & CrERF5 & MK862158 & Catharanthus roseus & [9] \\
\hline & CR1 & cra_locus_10963 & Catharanthus roseus & [10] \\
\hline & NtERF189 & AB827951 & Nicotiana tabacum & [11] \\
\hline & NtERF221/ORC1 & CQ808982 & Nicotiana tabacum & {$[12,13]$} \\
\hline & NtERF32 & AB828154 & Nicotiana tabacum & {$[14]$} \\
\hline & GAME9/JRE4 & Solyc01g090340 & Solanum lycopersicum & {$[15,16]$} \\
\hline & OpERF2 & LC171328 & Ophiorrhiza pumila & [17] \\
\hline & EcERF2 & Eca_sc006292.1_g0200.1 & Eschscholzia californica & [18] \\
\hline & EcERF3 & Eca_sc006292.1_g0150.1 & Eschscholzia californica & [18] \\
\hline & EcERF4 & Eca_sc006292.1_g0190.1 & Eschscholzia californica & [18] \\
\hline & EcERF12 & Eca_sc194641.1_g1370.1 & Eschscholzia californica & [18] \\
\hline \multirow{6}{*}{ WRKY } & CjWRKY1 & AB267401 & Coptis japonica & [19] \\
\hline & CrWRKY1 & HQ646368 & Catharanthus roseus & [20] \\
\hline & PsWRKY & JQ775582 & Papaver somniferum & [21] \\
\hline & OpWRKY1 & Opuchr09_g0007470-1 & Ophiorrhiza pumila & {$[22]$} \\
\hline & OpWRKY2 & Opuchr02_g0001210-1 & Ophiorrhiza pumila & [23] \\
\hline & OpWRKY3 & Opuchr09_g0002440-1 & Ophiorrhiza pumila & [24] \\
\hline \multirow{14}{*}{ bHLH } & NbbHLH1 & GQ859152 & Nicotiana benthamiana & [25] \\
\hline & NbbHLH2 & GQ859153 & Nicotiana benthamiana & [25] \\
\hline & NtMYC2a & HM466974 & Nicotiana tabacum & {$[26,27]$} \\
\hline & NtMYC2b & HM466975 & Nicotiana tabacum & {$[26,28]$} \\
\hline & CrMYC1 & AF283506 & Catharanthus roseus & [29] \\
\hline & CrMYC2 & AF283507 & Catharanthus roseus & [30] \\
\hline & SIMYC2 & NM_001324483 & Solanum lycopersicum & [15] \\
\hline & CrBIS1 & KM409646 & Catharanthus roseus & [31] \\
\hline & CrBIS2 & KM409645 & Catharanthus roseus & [32] \\
\hline & CrBIS3 & MN646782 & Catharanthus roseus & [33] \\
\hline & CjbHLH1 & AB564544 & Coptis japonica & [34] \\
\hline & EcbHLH1-1 & AB910896 & Eschscholzia californica & [35] \\
\hline & EcbHLH1-2 & AB910897 & Eschscholzia californica & [35] \\
\hline & RMT1 & KY851107 & Catharanthus roseus & [36] \\
\hline \multirow{2}{*}{ MYB } & BPF-1 & AJ251686 & Catharanthus roseus & {$[37,38]$} \\
\hline & OpMYB1 & LC076107 & Ophiorrhiza pumila & [39] \\
\hline \multirow{2}{*}{ bZIP } & GBF1 & AF084971 & Catharanthus roseus & [40] \\
\hline & GBF2 & AF084972 & Catharanthus roseus & [40] \\
\hline \multirow{3}{*}{ TFIIIA zinc finger } & ZCT1 & AJ632082 & Catharanthus roseus & [41] \\
\hline & $\mathrm{ZCT} 2$ & AJ632083 & Catharanthus roseus & [41] \\
\hline & ZCT3 & AJ632084 & Catharanthus roseus & [41] \\
\hline
\end{tabular}


Table 1. cont.

\begin{tabular}{|c|c|c|c|c|}
\hline Family & Name & Accession No. or Gene ID & Plant Species & Reference \\
\hline GATA & CrGATA1 & CRO_T134526 & Catharanthus roseus & [42] \\
\hline \multirow{5}{*}{ AT-hook } & 2D328 & EF025306 & Catharanthus roseus & [43] \\
\hline & 2D173 & EF025307 & Catharanthus roseus & [43] \\
\hline & 2D449 & EF025308 & Catharanthus roseus & [43] \\
\hline & $2 \mathrm{D} 38 \mathrm{M}$ & EF025309 & Catharanthus roseus & [43] \\
\hline & 2D7 & EF025310 & Catharanthus roseus & [43] \\
\hline
\end{tabular}

\subsection{APETALA2/Ethylene-Responsive Factor (AP2/ERF) TFs}

AP2/ERF family proteins form one of the largest groups of plant-specific TFs. The members contain an AP2/ERF domain that consists of approximately 60 amino acid residues and is involved in DNA-binding. Based on the number of AP2/ERF domains, the AP2/ERF superfamily can be divided into three subfamilies: AP2 (two AP2/ERF domains), RAV (one B3 domain in addition to one AP2/ERF domain), and ERF (one AP2/ERF domain) [44]. The ERF family can be further divided into the dehydrationresponsive element binding (DREB) protein subfamily, whose members are well known to be involved in drought tolerance, and the ERF subfamily, whose members are involved in various stress responses. The DREB and ERF subfamily proteins are known to bind to the dehydration-responsive element (e.g., GCCGAC) and the GCC box (e.g., AGCCGCC), respectively [45]. The AP2 and RAV family proteins are involved in plant growth and flower development, whereas the DREB and ERF subfamily proteins play an important role in biotic and/or abiotic stress responses, disease resistance, and plant hormone signaling. Jasmonate (JA)-responsive members of the ERF subfamily, which predominantly belong to the Group IX subgroup, play a key role in the biosynthesis of plant-specialized metabolites, including those of several alkaloids [3,17,18,46,47]. Group IX ERF genes frequently form gene clusters in nicotine, SGA, and MIA biosynthesis, which suggests the evolutionary significance of ERF clusters [48].

\subsection{WRKY TFS}

WRKY superfamily proteins are also found mainly in plants. Many WRKY TFs have been shown to play pivotal roles in plant development, senescence, and defense responses $[49,50]$. All the WRKY proteins contain at least one WRKY domain composed of approximately 60 amino acid residues, which includes a highly conserved WRKYGQK sequence at the $\mathrm{N}$-terminal and a zinc finger motif at the C-terminal [51]. The WRKY superfamily can be divided into three groups: Group I (two WRKY domains with a C2H2type zinc finger), Group II (one WRKY domain with a C2H2-type zinc finger), and Group III (one WRKY domain with a C2HC-type zinc finger). Group II WRKYs can be further classified into five subgroups: IIa, IIb, IIc, IId, and IIe. The WRKY domain typically binds to the W-box DNA sequence motif (e.g., TTGACT) [50]. Recent structural analyses revealed that the conserved WRKYGQK motif with a beta-sheet structure binds to the major groove of the DNA strand [52,53]. Several JA-responsive WRKY TF proteins belonging to different groups have been reported to be involved in the regulation of alkaloid biosynthesis, as well as that of other specialized metabolites $[2,3,22-24,54]$.

\subsection{Basic Helix-Loop-Helix (bHLH) TFs}

bHLH superfamily proteins are broadly distributed in eukaryotic kingdoms and are involved in many essential biological processes. In animals, bHLH TFs can be classified into six groups (A to F) that regulate circadian rhythm, cell cycle, and developmental processes $[55,56]$. In plants, bHLH proteins were first divided into 12 groups (Groups I to XII) based on the phylogenetic tree of $A$. thaliana bHLH TFs [57]. However, after sequencing the genomes of several plant and algal species, bHLH TFs were further classified into 32 subfamilies by means of phylogenetic analysis [58]. Plant bHLH TFs are involved in a wide 
array of physiological and developmental processes, including light and phytohormone signaling, biotic/abiotic stress responses, and tissue and organ development [59,60]. In addition, bHLH TFs have also been shown to regulate the biosynthesis of plant-specialized metabolites, including alkaloids $[2,3,46,61,62]$. The bHLH domain consists of approximately 60 amino acids, with two functionally different regions. The N-terminal end of the bHLH domain, which is composed of 15-20 amino acid residues in the basic region, is involved in DNA-binding, while two amphipathic alpha helices with a linking loop of variable lengths and sequences in the HLH region are involved in the formation of homodimeric or heterodimeric complexes. Based on their DNA-binding properties [55], most bHLH proteins in plants belong to Group B and specifically bind to the E-box (e.g., CANNTG).

MYC2, found in JA-insensitive jai1/jin1 mutants of $A$. thaliana, is a key component of the JA signaling pathway $[63,64]$. JA promotes the degradation of jasmonate ZIM domain (JAZ) repressor proteins by CORONATINE-INSENSITIVE 1 (COI1) and 26S proteasome and the release of MYC2 for the upregulation of JA-responsive genes. MYC2-type bHLH TFs also play an essential role in the regulation of specialized metabolism in many plant species [3,65-68], whereas non-MYC2-type bHLH TFs have been reported to control the biosynthesis of BIA and might be a type of bHLH protein in BIA-producing plant species [69]. In addition, subgroup IV bHLH TFs based on the phylogenetic analysis of AtbHLH proteins have been shown to regulate triterpene saponin and MIA biosynthesis in several plant species [31,70,71]. These JA-responsive MYC2-type and non-MYC2-type bHLH TFs orchestrate transcriptional changes in the alkaloid biosynthetic pathways.

\subsection{Other TFs}

MYB superfamily proteins are widely distributed in plants and play important roles in plant growth, development, and stress responses. MYB proteins contain an MYB DNAbinding domain composed of approximately 50 amino acid residues. The MYB domain generally comprises one to four imperfect repeats. Plant MYB TFs can be classified into four groups: R2R3-MYB, R1R2R3-MYB, 4R-MYB, and MYB-related proteins. Although many R2R3-MYB TFs have been reported to be involved in the regulation of specialized metabolites, such as glucosinolate, camalexin, and in flavonoid biosynthesis [3,72], only a few MYB TFs involved in the regulation of alkaloid biosynthesis have been reported thus far [37-39].

The basic region/leucine zipper (bZIP) superfamily is found in all eukaryotes and plays a critical role in defense and stress responses, phytohormone signaling, and senescence in plants. The bZIP proteins are typically divided into 10 groups (A-I and S) in A. thaliana. Two bZIP TFs have been identified in C. roseus and confirmed to bind to a G-box-like sequence. In addition, C2H2-type zinc finger proteins, GATA TF, and AT-hook motif nuclear localized TFs have also been reported.

\section{TFs Involved in the Biosynthesis of Universal Secondary Metabolites, Such as Phenylpropanoids and Terpenoids, in the Model Plant, Arabidopsis}

Many research groups have elucidated the regulatory mechanisms of phenylpropanoid and terpenoid biosynthesis, which are commonly found in almost all plant species. In the present study, we briefly describe several TFs that are involved in the regulation of phenylpropanoid, terpenoid, and camalexin biosynthesis in A. thaliana.

\subsection{TFs in the Phenylpropanoid Pathway}

In the biosynthesis of phenylpropanoids including anthocyanin (flower pigments), the complex that includes WD40-repeat (WD40) protein, Transparent Testa Glabra 1 (TTG1), R2R3-MYB TFs such as Production of Anthocyanin Pigment 1 (PAP1)/MYB75, PAP2/MYB90, MYB113, and MYB114, and bHLH TFs such as Glabra 3 (GL3), Enhancer of GL3 (EGL3), and Transparent Testa 8 (TT8) acts as an important regulatory module and regulates the expression of the "late" anthocyanin biosynthetic enzyme genes in $A$. thaliana [61,73]. This MYB-bHLH-WD40 (MBW) complex is widely distributed in phenylpropanoid biosynthesis [74] (Figure 1A). 
A

\section{Phenylpropanoid biosynthesis}

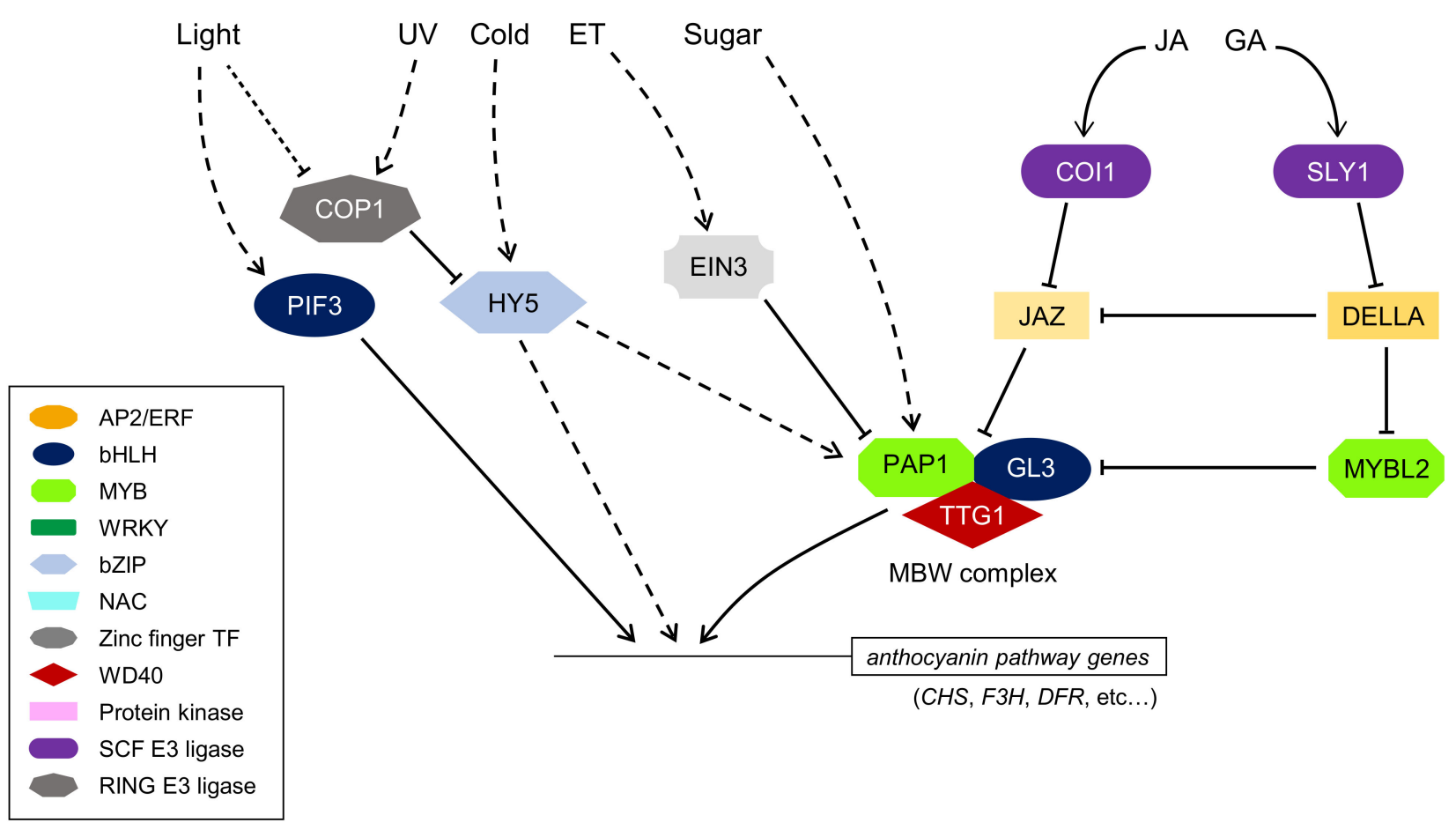

\section{B}

\section{Terpenoid biosynthesis}

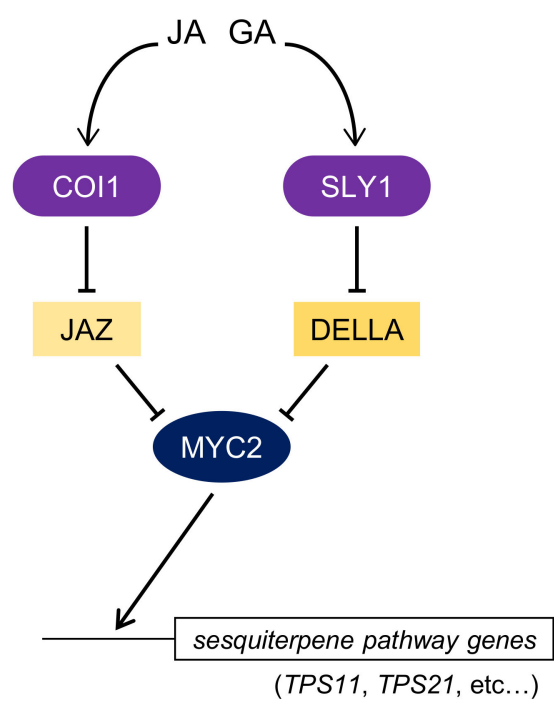

\section{Alkaloid biosynthesis}

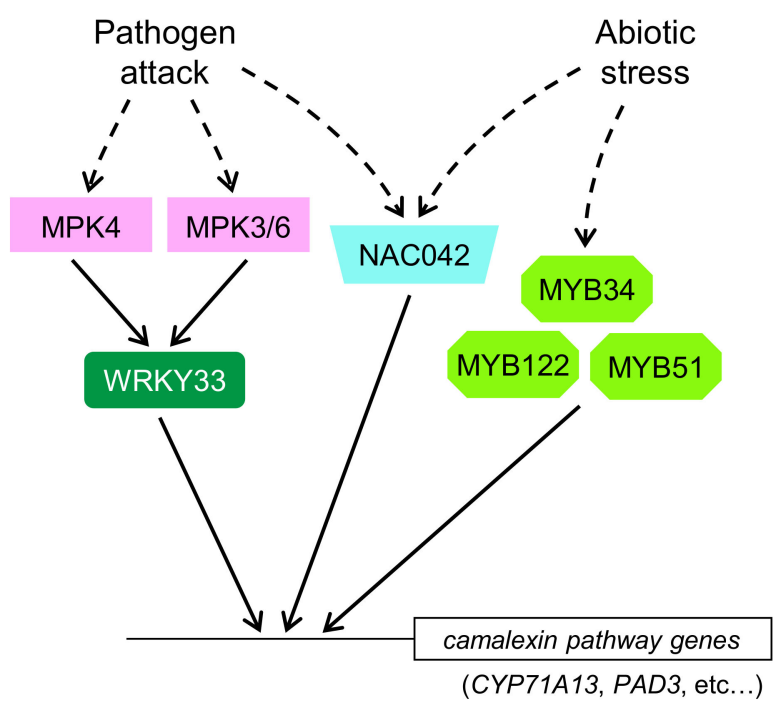

Figure 1. A brief model of the regulation of phenylpropanoid (A), terpenoid (B), and camalexin $(\mathbf{C})$ biosynthesis in A. thaliana. Black arrows indicate upregulation, while T-shaped lines indicate inhibition. Broken lines show indirect or undetermined steps that are possibly involved in uncharacterized proteins or unidentified regulation. JAZ and DELLA include the functionally redundant proteins. Abbreviations: CHS, Chalcone synthase; COI1, Coronatine-insensitive 1; COP1, Constitutive photomorphogenesis 1; DFR, Dihydro flavonol reductase; EIN3, Ethylene-insensitive 3; ET, Ethylene; F3H, Flavanone 3-hydroxylase; GA, Gibberellin; GL3, Glabra 3; HY5, Elongated Hypocotyl 5; JA, Jasmonate; JAZ, Jasmonate ZIM domain; MBW, MYB-bHLH-WD40; MYBL2, MYB-LIKE2; PAD3, Phytoalexin-deficient 3; PAP1, Production of Anthocyanin Pigment 1; PIF3, Phytochrome-interacting factor 3; TPS, terpene synthase; TTG1, Transparent Testa Glabra 1; SLY1, Sleepy 1. 
Developmental signals and environmental stresses, such as sugar, UV light, temperature, and hormone signaling, are known to control anthocyanin biosynthesis. For instance, sucrose-induced anthocyanin biosynthesis depends on the function of PAP1/MYB75 [75]. Gibberellin (GA) represses sucrose-induced anthocyanin production because it induces the degradation of DELLA proteins, which are negative regulators of GA signaling, and the interaction of MYB-LIKE2 (MYBL2) with TT8, resulting in the suppression of anthocyanin biosynthesis [76,77]. JA is also involved in sucrose-induced anthocyanin biosynthesis, mediated by the interaction of JAZ proteins with the MBW complex $[76,78,79]$, suggesting that the COI1-JAZ receptor complex may be conserved in many plant species and involved in the biosynthesis of various secondary metabolites, as discussed below. Ethylene (ET) inhibits sugar- and light-induced anthocyanin production [80]. ETHYLENE-INSENSITIVE 3 (EIN3), a key factor in ET signaling, directly suppresses the expression of TT8 [81]. High light, UV light, or cold stress also induces anthocyanin biosynthesis via the MBW complex or ELONGATED HYPOCOTYL 5 (HY5) TF [82-85]. A brief model of signal transduction involved in anthocyanin biosynthesis is described in Figure 1A.

\subsection{TFs in the Terpenoid Pathway}

Terpenoids synthesized from isoprene (C5) units also play diverse roles in plantenvironment interactions [86]. AtMYC2-mediated positive regulation of the biosynthesis of these sesquiterpenes, the so-called phytoalexins, has been reported [87]. Methyl jasmonate (MeJA) and GA significantly increase the expression of the terpenoid pathway genes. AtMYC2 controls sesquiterpene biosynthesis in a JA-responsive manner through its interaction with GA signaling, especially through the competitive interaction of JAZ and DELLA in the JA and GA signaling cascades (Figure 1B).

\subsection{TFs in the Camalexin Pathway}

A. thaliana also produces an indole alkaloid, camalexin, as phytoalexin [88]. AtWRKY33 plays a crucial role in the biosynthesis of camalexin in response to pathogen attacks via the signaling cascade of mitogen-activated protein kinases (MAPKs) [89]. ANAC042, a member of the NAM, ATAF1/2, and CUC2 (NAC) TF family proteins, and MYB proteins have been reported to be involved in camalexin biosynthesis [72,90] (Figure 1C). The unique feature of camalexin biosynthesis is that the expression of camalexin biosynthetic genes is restricted to the tissue undergoing cell death [91]. The findings that cerato-platanin protein (an elicitor) produced by Ceratocystis platani triggers salicylic acid (SA)- and ET-signaling pathways, but not the JA signaling pathway, and induces the biosynthesis of camalexin in A. thaliana, indicated that camalexin biosynthesis is independent of JA signaling [92], whereas alkaloid biosynthesis in other pathways is largely dependent on JA signaling, as discussed below.

\section{TFs Involved in The Regulation of Alkaloid Biosynthetic Enzyme Genes Expression}

Alkaloid biosynthesis is regulated by several TF family proteins, such as bHLH and MYB, which are also found in the regulation of phenylpropanoid and terpenoid biosynthesis in A. thaliana; however, different types (groups) of TFs have been reported in several alkaloid-producing plant species. In this section, we describe TFs involved in the regulation of nicotine, SGA, MIA, and BIA biosynthesis.

\subsection{TFs in the Nicotine and SGA Pathways}

Nicotine is biosynthesized in the Nicotiana species by means of pyrrolidine ring formation and pyridine ring formation, followed by coupling of both rings [1]. Ornithine or arginine is converted to putrescine, which is then converted to $N$-methylputrescine, the first specific metabolite for the biosynthesis of nicotine, by putrescine $\mathrm{N}$-methyltransferase (PMT). Aspartate is converted to nicotinic acid containing the pyridine ring by several enzymes, including quinolinic acid phosphoribosyltransferase (QPT). Although the mechanism of final condensation to produce nicotine is not enzymatically revealed, A622, a 
pinoresinol-lariciresinol reductase/isoflavone reductase/phenylcoumaran benzylic ether reductase family oxidoreductase, is required for the coupling.

To explore the TFs involved in nicotine biosynthesis, the cDNA-amplified fragment length polymorphism approach, in combination with target metabolite analysis, was performed using JA-treated tobacco Bright Yellow-2 (BY-2) cultured cells [93]. In these studies, two AP2/ERF genes, NtORC1/ERF221 and NtJAP1/ERF10, were identified [12,94]. NtORC1/ERF221 and NtJAP1/ERF10 positively regulated the PMT gene, while the overexpression of NtORC1/ERF221 only resulted in high nicotine alkaloid [13].

$A P 2 / E R F$ genes were independently identified using microarray analysis of a Nicotiana tabacum NIC2 mutant [11]. The NIC2 locus includes seven clustered AP2/ERF genes belonging to the group IX AP2/ERF subfamily. Among them, the NtERF189 gene, which encodes for subclade 2-1 of group IX AP2/ERF protein, showed clear responsiveness to $\mathrm{MeJA}$ and was expressed in roots, where nicotine is preferentially produced. RNA silencing of NtERF189 significantly reduced the expression levels of nicotine biosynthetic enzyme genes, including PMT and QPT. The overexpression of NtERF189 also showed a marked increase in the transcript levels of these genes, suggesting that NtERF189 functions as a master regulator of nicotine biosynthesis in tobacco (Figure 2A). Recently, the overexpression of NtERF189 in stable transgenic tobacco plants has been analyzed [95]. These plants showed an increase in the total alkaloid content in leaves, up to 4.3- to 17.5-fold; in addition, the plant growth was also affected, possibly due to the drastic alteration in the contents of nitrogen-containing compounds. The NtERF189 protein recognizes GCC-box-like elements, $(\mathrm{A} / \mathrm{C}) \mathrm{GC}(\mathrm{A} / \mathrm{C})(\mathrm{A} / \mathrm{C}) \mathrm{NCC}$, in the promoter of nicotine biosynthetic enzyme genes [96]. Interestingly, NtERF189-binding sites were found in the promoter of the MeJA-responsive QPT2 gene involved in nicotine biosynthesis, but not in that of the QPT1 gene which is not induced by MeJA. This suggests that ERF189-binding sites might have been acquired due to gene duplication in the QPT2 promoter during evolution [97].

Another AP2 / ERF gene, NtERF32 (EREBP2), has also been identified in tobacco plants. This gene encodes the group IX AP2/ERF protein, which is not a part of the NIC2 locus. NtERF32 might function as a transcriptional activator to regulate the expression of multiple genes involved in nicotine biosynthesis by binding to the GCC-box-like element [14].

Several groups have reported that MYC2-type bHLH TFs are involved in the regulation of nicotine biosynthesis. Todd et al. performed functional screening and identified two MeJA-responsive bHLH TF genes, NbbHLH1 and NbbHLH2, in Nicotiana benthamiana [25]. RNA silencing of $\mathrm{NbbHLH1}$ and $\mathrm{NbbHLH} 2$ significantly decreased the expression levels of nicotine biosynthetic enzyme genes, whereas their overexpression slightly increased PMT expression and nicotine content. NbbHLH1 can bind to the G-box in the PMT promoter, which contains the target GCC-box of NtORC1/ERF221 proximal to this G-box. NbbHLH1 and NtORC1/ERF221 cooperatively regulate the expression of PMT by interacting with both the elements. NbbHLH1 interacts with AtJAZ1 and NtJAZ1, suggesting that JA-mediated regulation of nicotine biosynthesis happens through the MYC2-JAZ complex [13].

Highly homologous genes of NbbHLH1 and NbbHLH2 have also been identified in $N$. tabacum. NtMYC2a and NtMYC2b positively regulate the transcript levels of nicotine biosynthetic enzymes and transporter genes as well as NIC2-locus AP2/ERF genes [26-28]. The direct interaction between NtMYC2 and NtJAZ1 has also been confirmed. NtMYC2 forms a regulatory complex with NtJAZ1 and controls the expression of both nicotine biosynthesis genes and other $A P 2 / E R F$ genes in JA signaling.

SGAs are commonly produced by plants belonging to the Solanaceae family. The biosynthetic pathway starts with the precursor cholesterol and proceeds via hydroxylation, oxidation, glycosylation, and transamination steps to generate various SGAs [1]. The genomes of tomato and potato revealed that clustered genes named glycoalkaloid metabolism $(G A M E)$ are involved in the central pathway of SGAs. 
A

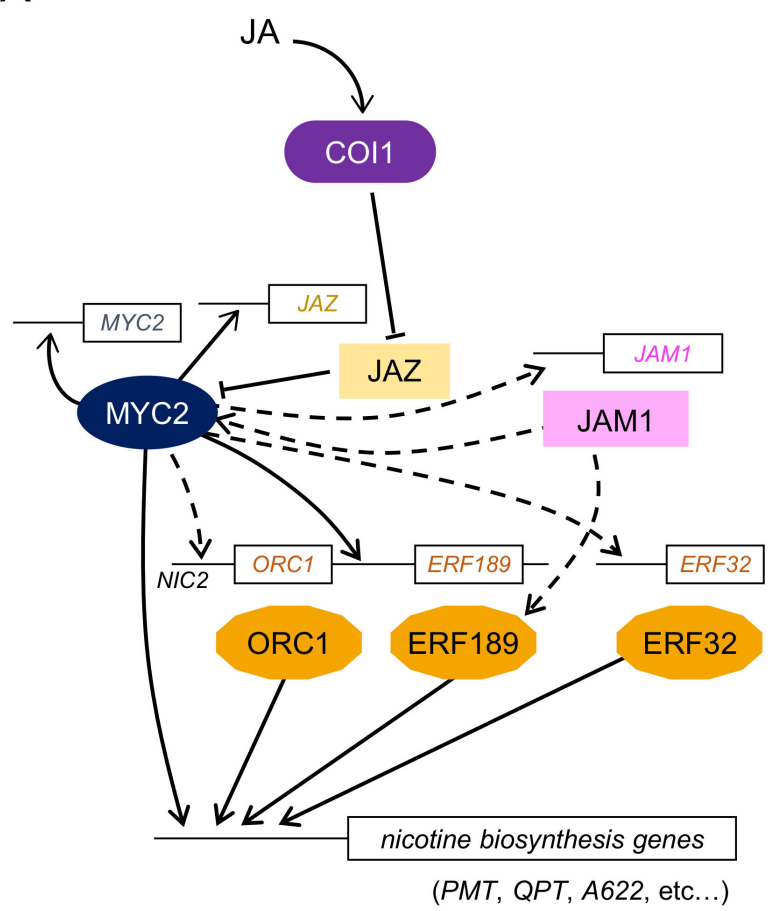

B

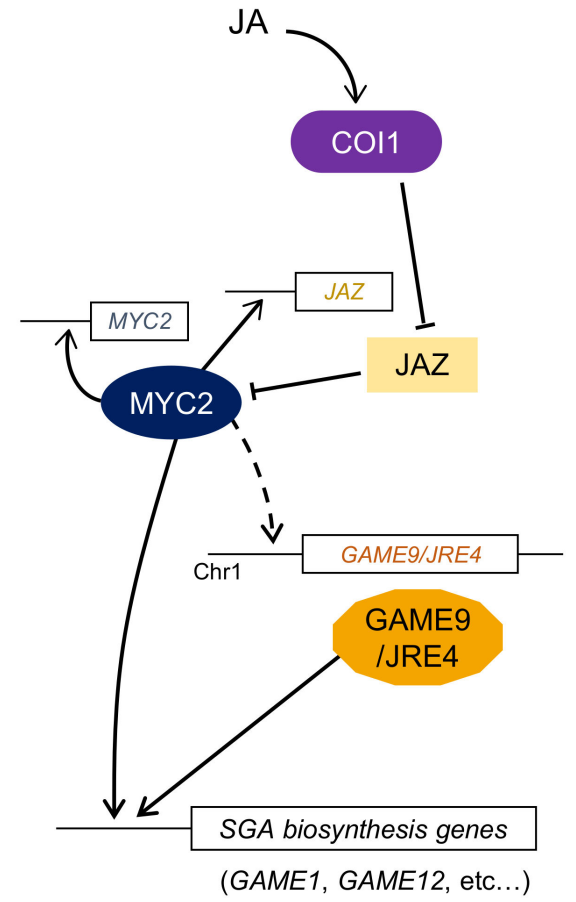

Figure 2. A simplified model of the transcriptional network of JA signaling in nicotine biosynthesis in tobacco (A) and SGA biosynthesis in tomato (B). Boxes with straight lines indicate regulator genes, including promoter regions. Black arrows indicate upregulation, while T-shaped lines indicate inhibition. Broken lines show indirect or undetermined steps that are possibly involved in uncharacterized proteins or unidentified regulation. Possible feedback regulation via MYC2 TF, which is known to regulate the expression of several JAZ genes, is shown. In tobacco, functionally redundant MYC2 proteins such as NtMYC2a, NtMYC2b, NbbHLH1, and NbbHLH2 have been identified; of these, they are collectively indicated with MYC2. ORC1 and ERF189 genes and GAME9/JRE4 are clustered in the NIC2 locus in N. tabacum and chromosome 1 in tomato, respectively, with other group IX AP2 /ERF genes. Abbreviations: GAME, Glycoalkaloid metabolism; JAM1, JA-factor-stimulating MAPKK1; JRE4, JA-responsive ethylene response factor 4; PMT, Putrescine N-methyltransferase; QPT, Quinolinic acid phosphoribosyltransferase; SGA, Steroidal glycoalkaloid.

Two research groups identified a novel AP2/ERF transcription factor, GAME9/JAresponsive ethylene response factor 4 (JRE4), which is involved in the regulation of SGA biosynthesis in tomato and potato $[15,16]$. GAME9/JRE4 was co-expressed with 37 genes involved in SGA biosynthesis and was highly expressed in leaves, buds, and immature green fruit. SIGAME9-RNA interference (RNAi) and jre4_1 mutant tomato plants showed decreased SGA content and transcript levels of biosynthetic enzyme genes $[15,98]$. Overexpression of GAME9 altered the expression of genes involved in sterol and SGA biosynthesis (Figure 2B). Although GAME9/JRE4 was clustered with four homologous genes, only GAME9/JRE4 was confirmed to control SGA biosynthesis. Furthermore, SIMYC2 and SIGAME9/JRE4 might bind to the GCC-box and G-box in the proximal region of promoters and coordinately regulate SGA biosynthesis genes in tomato. Such synergistic regulation of pathway genes by group IX AP2/ERF and MYC2 TFs is shared in nicotine and SGA biosynthesis.

\subsection{TFs in the MIA Pathway}

MIAs are biosynthesized by means of two primary metabolic routes: the shikimate and methylerythritol phosphate (MEP) pathways [1]. Tryptamine derived from tryptophan and secologanin derived from geranyl diphosphate (GPP) are condensed to strictosidine, the central intermediate of MIAs, by strictosidine synthase (STR). Strictosidine is deglucosylated by strictosidine glucosidase (SGD) and then converted to diverse MIAs, such as 
serpentine, catharanthine, vindoline, and anti-tumor bisindole alkaloids (vinblastine and vincristine).

The first alkaloid TFs to be identified in C. roseus using yeast one-hybrid screening were AP2/ERF TFs, which were named octadecanoid-derivative responsive Catharanthus AP2-domain (ORCA) 1 and ORCA2 [4]. ORCA1 binds to the JA-responsive element (JRE) of the STR promoter but has little transcriptional activity, whereas ORCA2 not only binds to the JRE but also shows in vivo trans-activation activity and JA-responsive expression. The overexpression of ORCA2 in hairy roots also suggests a key function of ORCA2 in MIA biosynthesis [99]. ORCA3, another MeJA-responsive AP2/ERF TF, was identified in C. roseus using an activation-tagging approach $[5,100]$. ORCA3 induces the expression of several MIA biosynthetic genes, including STR. Hence, ORCA3 is considered a master regulator in MIA biosynthesis, but it is not sufficient for the complete regulation of the MIA biosynthetic pathway (Figure 3).

More recently, ORCA4, ORCA5, and ORCA6 have been reported to be present in the same genomic region as ORCA2 and ORCA3 [6-8]. All clustered ORCA genes were upregulated in response to MeJA, but their expression patterns were slightly different. Using transient reporter assay, ORCA4, ORCA5, and ORCA6 were found to trans-activate the STR promoter, similar to ORCA2 and ORCA3. Overexpression of ORCA4 and ORCA5 in C. roseus hairy roots and transient overexpression of ORCA6 in C. roseus flower petals resulted in the upregulation of MIA biosynthetic enzyme genes as well as other TF genes, including ORCA genes, and a significant increase in the accumulation level of several MIAs. Clustered ORCA TFs regulate the expression of different sets of MIA biosynthetic genes, suggesting that their function might overlap and diversify during evolution.

In addition to ORCA TFs, a few AP2/ERF TFs have also been identified in C. roseus. CrERF5 belongs to a different clade from ORCA TFs in the phylogenetic tree and activates the MIA biosynthetic pathway, especially the bisindole alkaloid pathway [9]. The expression of CrERF5 was induced in response to MeJA and ET, suggesting that ET signaling might affect the production of bisindole alkaloids. Catharanthus roseus 1 (CR1) was also identified in C. roseus and characterized as a transcriptional repressor of MIA biosynthesis. The expression of $C R 1$ decreased in response to MeJA, and the suppression of $C R 1$ showed an increase in the accumulation of serpentine and vindoline [10]. These AP2/ERF genes were characterized using virus-induced gene silencing (VIGS), an effective tool for the investigation of gene function.

CrMYC1 was the first bHLH TF to be reported for MIA biosynthesis [29]. Although the binding activity of CrMYC1 to the G-box sequence in the STR promoter was confirmed, there was no further characterization to show that CrMYC1 plays a role in the regulation of MIA biosynthesis in C. roseus. Zhang et al. have identified another bHLH TF, CrMYC2, in C. roseus [30]. CrMYC2 is homologous to AtMYC2 and regulates the expression of $O R C A 2$ and $O R C A 3$, leading to the regulation of MIA biosynthesis. It also regulates the expression of $\mathrm{CrJAZ}$ genes and directly interacts with $\mathrm{CrJAZ}$ proteins that repress the function of CrMYC2 [36]. Upon overexpression of $C r M Y C 2$, the expression levels of ORCA4 and ORCA5 were also upregulated; however, CrMYC2 could not directly trans-activate the ORCA4 and ORCA5 promoters, suggesting that unknown TFs might play a role in regulating the expression of these genes [6]. While CrMYC2 directly binds to the promoter of the tryptophan decarboxylase (TDC) gene, a key gene for tryptamine biosynthesis, the expression of STR is indirectly regulated via ORCA3.

Based on the classification of plant bHLH proteins, MYC2 belongs to group IIIe [57]. Recently, non-MYC2 type bHLH proteins, which belong to group IVa, have been identified in C. roseus and designated as bHLH iridoid synthesis (BIS) [31]. BIS1, BIS2, and BIS3 genes are clustered in the same genomic region of $C$. roseus, and their expression can be induced using MeJA. BIS1 and BIS2 form homo- or heterodimers, while a clear interaction with CrMYC2 has not been observed. BIS proteins regulate the expression of genes involved in the secoiridoid pathway [31-33]. 


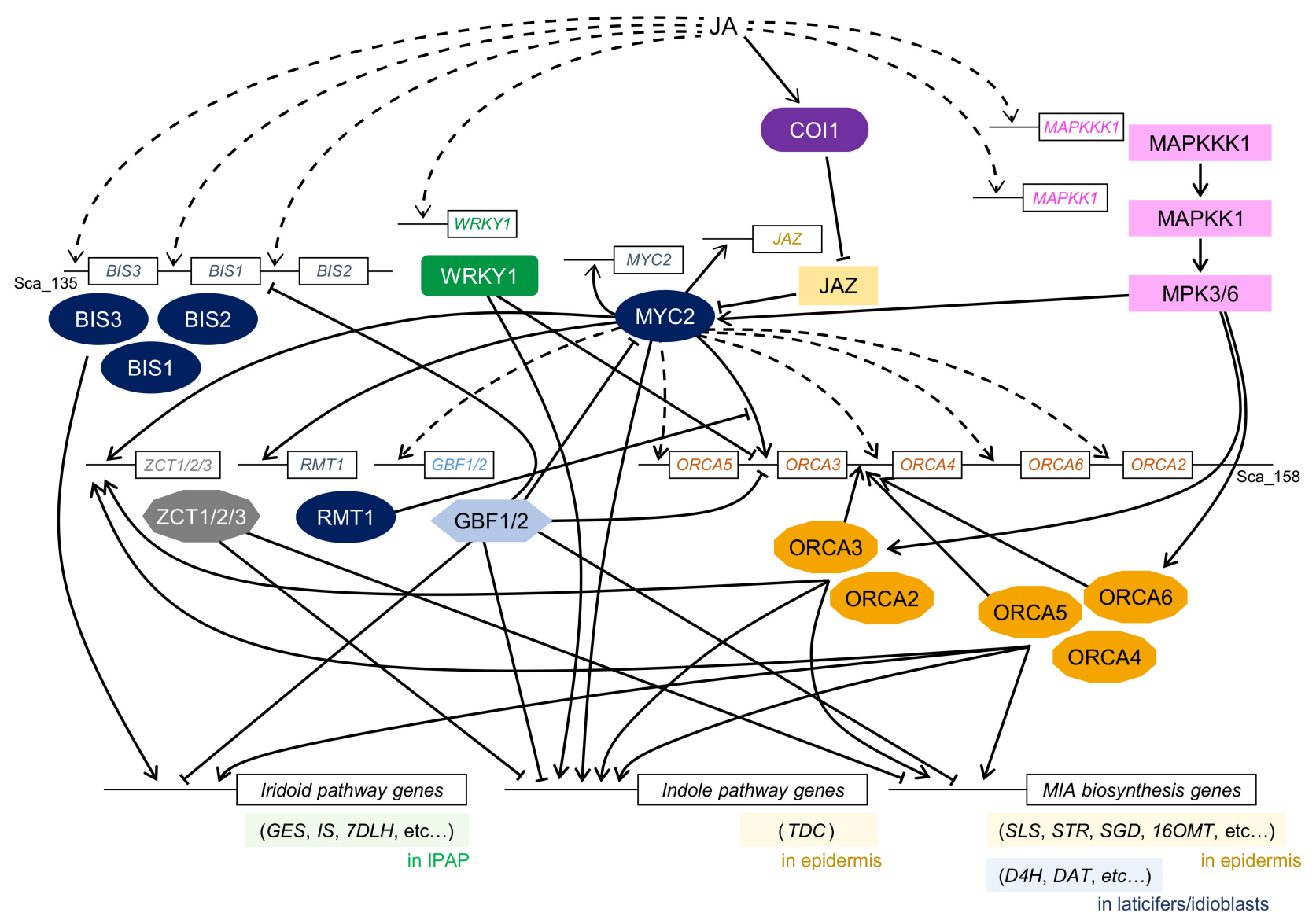

Figure 3. A simplified model of the transcriptional network of JA signaling involved in MIA biosynthesis in C. roseus. Boxes with straight lines indicate regulator genes, including promoter regions. Black arrows indicate upregulation, and T-shaped lines indicate inhibition. Broken lines show indirect or undetermined steps possibly involved in uncharacterized proteins or unidentified regulation. GES, IS, and 7DLH marked in the green box work in the iridoid pathway to synthesize loganic acid in internal phloem-associated parenchyma (IPAP), while D4H and DAT marked in the blue box function in the biosynthesis of vindoline in laticifers/idioblasts. The relationship between tissue/cell-specific regulation of enzyme genes and JA-responsive TFs remains unknown. Secologanin, tryptamine, strictosidine, tabersonine, and desacetoxyvindoline are synthesized in the upper or lower epidermis by SLS, STR, SGD, 16OMT, and TDC marked in the yellow boxes. Possible feedback regulation via MYC2 TF, which is known to regulate the expression of its own and JAZ genes, is shown. It is still unknown whether the expression of other genes such as BIS, WRKY1, and MAPKKK1 is directly regulated by MYC2. $B I S$ and ORCA genes are clustered in the same C. roseus genome scaffolds, scaffold_135 and scaffold_158, respectively. Abbreviations: BIS, bHLH iridoid synthesis; DAT, Deacetylvindoline 4-O-acetyltransferase; D4H, Desacetoxyvindoline 4-hydroxylase; GBF, G-box binding factor; GES, Geraniol synthase; IS, Iridoid synthase; MIA, Monoterpenoid indole alkaloid; ORCA, Octadecanoid-derivative responsive Catharanthus AP2-domain; RMT, Repressor of MYC2 Target 1; SGD, Strictosidine glucosidase; SLS, Secologanin synthase; STR, Strictosidine synthase; TDC, Tryptophan decarboxylase; 16OMT, 16-hydroxytabersonine O-methyltransferase; 7DLH, 7-deoxyloganic acid hydroxylase.

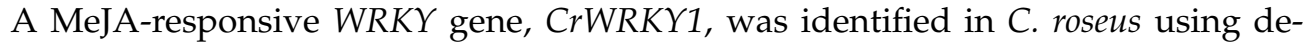
generate PCR [20]. CrWRKY1 belongs to the Group III subfamily, and overexpression of $C r W R K Y 1$ caused an increase in the expression of TDC gene and decrease in those of ORCA2 and ORCA3 genes, thus suggesting that CrWRKY1 might preferentially regulate serpentine biosynthetic pathway by activating TDC as well as repressing ORCA genes.

CrBPF-1 gene, which encodes a MYB-like box-P binding factor protein, was identified using yeast one-hybrid screening with the STR promoter region containing an elicitorresponsive element [37]. Since CrBPF-1 gene was significantly induced by an elicitor, but not MeJA, CrBPF-1 might function through JA-independent elicitor signaling. Overexpression 
of CrBPF-1 significantly upregulated the expression of MIA biosynthesis genes while modestly affecting the accumulation of metabolites [38].

Vom Endt et al. identified five DNA-binding AT-hook motif-containing proteins in C. roseus. AT-hook proteins could trans-activate the expression of the ORCA3 promoter, whereas the expression of $A T$-hook genes was not altered upon MeJA treatment, indicating that AT-hook proteins may act as activators independent of JA signaling [43].

Light is an important environmental cue for converting tabersonine to vindoline in C. roseus. Recently, light-induced GATA-type TF, CrGATA1, was identified and confirmed to be related to the alteration of gene expression involved in vindoline production in response to light [42]. Furthermore, the expression of $\mathrm{CrGATA1}$ is negatively controlled by the light-responsive phytochrome-interacting factor (PIF) family TF, CrPIF1.

Transcriptional repressors have also been identified in C. roseus and are considered to be involved in the modulation of MIA biosynthesis (Figure 3). Two G-box binding factor (GBF) proteins, CrGBF1 and CrGBF2, both containing the bZIP motif, possibly act as transcriptional repressors of MIA biosynthesis [40]. When GBF1 and GBF2 genes were co-expressed with $\mathrm{CrMYC2}$, these proteins directly interacted with $\mathrm{CrMYC2}$, suggesting that $\mathrm{CrGBF}$ proteins possibly act as antagonists of CrMYC2 and fine-tune gene expression in MIA biosynthesis [101].

Pauw et al. identified three genes that encode TFIIIA-type zinc finger proteins and named them ZCT1, ZCT2, and ZCT3, for zinc finger Catharanthus transcription factor [41]. RNA silencing of ZCT1 had little effect on the expression of MIA biosynthetic enzyme genes and production of MIA, indicating that the three ZCT repressors might function redundantly [102]. ZCT proteins act as transcriptional repressors of STR and TDC promoters. Since CrMYC2 and ORCA proteins activate the expression of ZCT genes [6-8,101], ZCT proteins might fine-tune the spatiotemporal expression of genes involved in MIA biosynthesis.

In A. thaliana, MYC2-like group IIId bHLH TFs are known to repress the expression of MYC2-target genes. The Repressor of MYC2 Target 1 (RMT1) was identified in C. roseus and confirmed to repress the expression of ORCA3 [36]. The expression of RMT1 was induced by MeJA and CrMYC2. RMT1 did not interact with CrMYC2 but directly bound to the AACGTG sequence in the ORCA3 promoter, which is a target of MYC2, suggesting that RMT1 represses the ORCA3 gene by competing with CrMYC2 to bind to the same cis-element.

Although several TFs have been characterized in the MIA pathway, the characterization of TFs involved in cell differentiation is limited. Since vindoline biosynthesis occurs in laticifer/idioblast cells, understanding the TFs involved in laticifer/idioblast differentiation is crucial for the production of vindoline in undifferentiated cells. Several TFs involved in laticifer differentiation have been characterized in Hevea brasiliensis; these include MYB TFs (identified using transcriptome analysis) and an AP2/ERF TF, HbEREBP1 as a negative regulator of defense genes in laticifers $[103,104]$.

\subsection{TFs in the BIA Pathway}

BIA biosynthesis starts with the conversion of L-tyrosine to dopamine and 4-hydroxyphenylacetaldehyde, followed by condensation to (S)-norcoclaurine, the central precursor of BIAs. (S)-norcoclaurine is converted to $(S)$-reticuline, the central intermediate of BIAs, by three methyltransferases and one cytochrome P450, and (S)-reticuline is converted to various BIAs such as analgesic morphine, antimicrobial berberine, and sanguinarine [1,105]. Almost all of the enzyme genes involved in the biosynthesis of these alkaloids have been identified and characterized, in addition to which metabolic engineering approaches have been attempted to manipulate the BIA biosynthetic pathways [1]. Furthermore, whole-genome sequencing of several BIA-producing plants such as P. somniferum, E. californica, Aquilegia coerulea, Nelumbo nucifera, Macleaya cordata, and Coptis chinensis has been performed, which provides us with more information for the identification of unknown genes involved in BIA biosynthesis [106-112]. 
A group IIc WRKY TF, CjWRKY1, was first identified in C. japonica using transient RNAi screening as a transcriptional activator in berberine biosynthesis [19] (Figure 4). The CjWRKY1 gene showed clear MeJA-responsiveness and CjWRKY1 regulated almost all BIA biosynthetic enzyme genes by binding to the $\mathrm{W}$-box in the promoter of target genes in C. japonica cells. Heterologous expression of CjWRKY1 in E. californica (California poppy), which shares a common BIA biosynthetic pathway, resulted in increased BIA production, although the expression levels of several BIA biosynthetic enzyme genes were not altered [113]. A recent genome-wide analysis of California poppy WRKY genes suggested that the WRKY TF family might be functionally diversified in BIA biosynthesis and associated with the accumulation and translocation of BIAs [114].

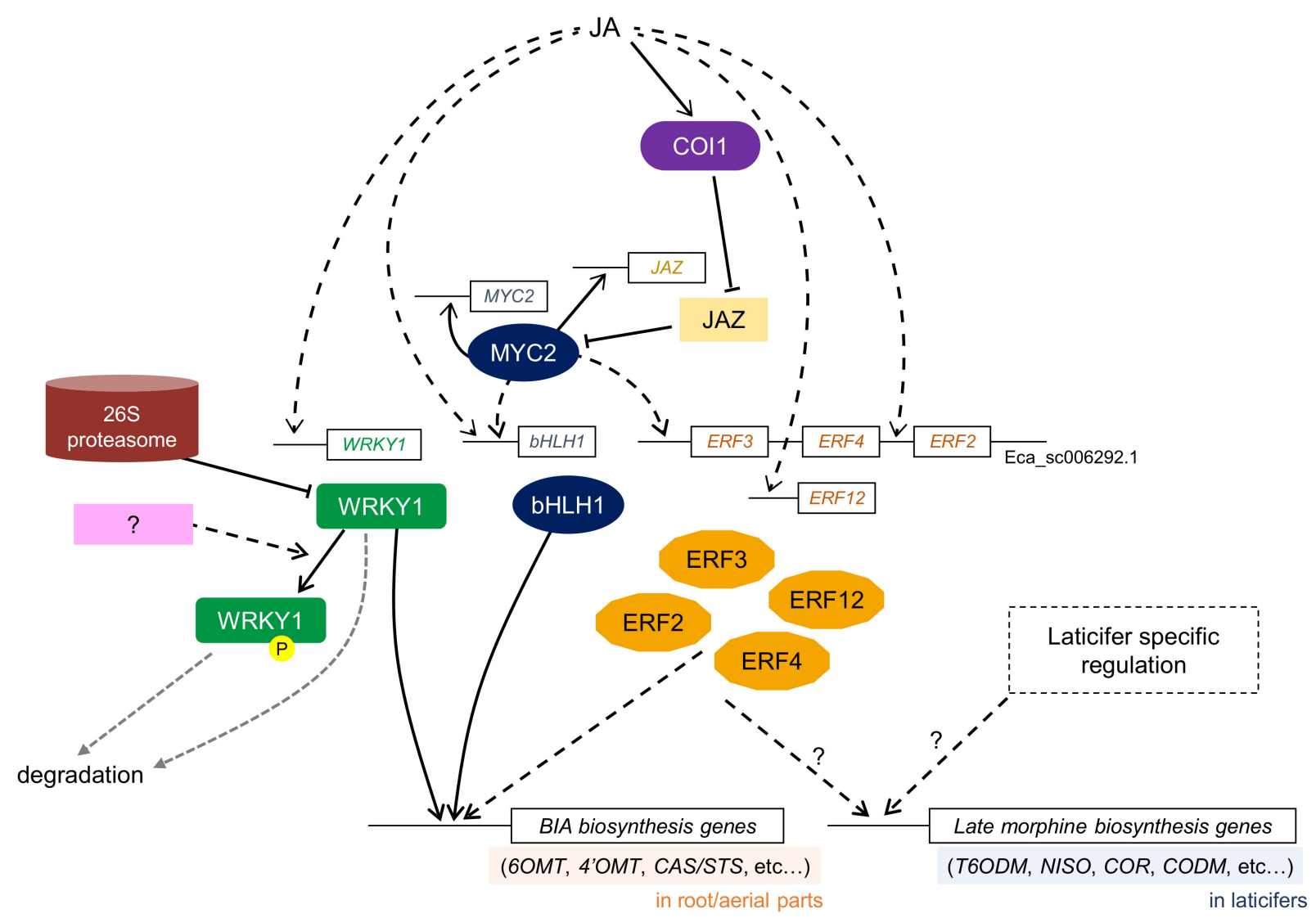

Figure 4. A simplified model of the transcriptional network of JA-signaling in BIA biosynthesis in C. japonica cultured cells and E. californica roots and cultured cells. Boxes with straight lines indicate regulator genes, including promoter regions. Black arrows and T-shaped lines indicate upregulation and inhibition, respectively. Broken lines show indirect or undetermined steps that are possibly involved in uncharacterized proteins or unidentified regulation. Gray dashed lines indicate degradation steps. Biosynthetic enzyme genes involved in late morphine pathway such as T6ODM, NISO, COR, and CODM might be controlled by laticifer-specific regulation, which remains to be determined. Since the functions of COI1, JAZ, and MYC2 proteins in BIA biosynthesis are not fully characterized, it is still unknown whether the expression of WRKY1, bHLH1, and several ERF genes is regulated by MYC2. Unphosphorylated WRKY1 is degraded by 26S proteasome, while unknown tyrosine kinase(s) and protease(s) might be involved in the phosphorylation and degradation of WRKY1, respectively, in C. japonica. BIA biosynthesis is regulated by several non-MYC2-type bHLH TFs (CjbHLH1 in C. japonica and EcbHLH1-1/2 in E. californica), while only bHLH1 is described here. ERF2, ERF3, and ERF4 genes are clustered in the same E. californica genome scaffold, Eca_sc006292.1. Abbreviations: BIA, Benzylisoquinoline alkaloid; CAS/STS, (S)canadine synthase/(S)-stylopine synthase; CODM, Codeine O-demethylase; COR, Codeinone reductase; NISO, Neopinone isomerase; T6ODM, Thebaine 6-O-demethylase; 6OMT, (S)-norcoclaurine 6-O-methyltransferase; 4'OMT, (S)-3'-hydroxy- $N$ methylcoclaurine 4'-O-methyltransferase. 
In P. somniferum, wound-induced PsWRKY, which belongs to the group I WRKY protein, was found to be involved in the BIA biosynthetic pathway [21]. PsWRKY can bind to the W-box DNA sequence and trans-activate the tyrosine decarboxylase (TYDC) gene promoter. Apuya et al. revealed that heterologous expression of A. thaliana WRKY1 (AtWRKY1), which also belongs to the group I WRKY protein, enhanced the production of BIAs in P. somniferum and E. californica cells [115]. These results indicate that group I WRKY TFs might also be involved in the regulation of BIA biosynthesis.

Transient RNAi screening in C. japonica cells further highlighted the bHLH TF, CjbHLH1, as a comprehensive transcriptional activator in BIA biosynthesis [34]. While MYC2-type bHLH TFs regulate the biosynthesis of nicotine and MIA, CjbHLH1 is a non-MYC2-type bHLH TF, as deduced based on the clear difference in amino acid sequence and length [69]. Interestingly, homologous proteins of CjbHLH1 are only found in BIA-producing plant species, and EcbHLH1-1/1-2, which are CjbHLH1 homologs of California poppy, have been confirmed to regulate BIA biosynthesis in California poppy cells [35]. These results suggest that a unique type of bHLH TF plays a key role in the regulation of BIA biosynthesis in BIA-producing plants, and functionally diverse bHLH TFs form different transcriptional networks in the biosynthesis of different types of alkaloids. Although the expression of CjbHLH1 and EcbHLH1-1/1-2 is induced by MeJA, the functional relationship between non-MYC2-type bHLH and MYC2 remains unclear.

Characterization of cis-acting elements in the promoter region of BIA biosynthetic enzyme genes in C. japonica cells indicated the involvement of AP2/ERF TF protein(s) in the regulation of BIA biosynthesis [116]. Genome-wide investigation of AP2/ERF family genes in the E. californica genome revealed that several Group IX EcAP2/ERF genes show a clear response to MeJA. Furthermore, four Group IX AP2/ERF TFs trans-activate BIA biosynthetic enzyme genes [18].

As discussed in the MIA pathway, morphine biosynthesis also occurs among several different cell types, including sieve elements and laticifer cells. The spatial accumulation of specific types of BIAs in opium poppy plants and cultured cells may be regulated by different networks of TFs. Furthermore, different organs produce different types of BIAs; the aerial part produces pavine-type BIA, while roots produce benzophenanthridinetype BIA in California poppy [114]. These organ-specific regulatory mechanisms of BIA biosynthesis have not been sufficiently characterized.

\section{Regulatory Mechanism: Upstream Signals, i.e., JA-Mediated, and Post-Transcriptional Regulation}

JAs are key signaling molecules involved in secondary metabolism, including alkaloid biosynthesis. As mentioned above, the JA signaling pathway has been intensively investigated in A. thaliana. MYC2, COI1, and JAZ form a regulatory complex with novel interactor of JAZ (NINJA), TOPLESS (TPL), and MEDIATOR 25 (MED25), and orchestrate the JA-signaling cascade; some homologs in alkaloid pathways have been isolated based on sequence homology [62].

Tobacco COI1 homologs, NaCOI1 and NtCOI1, have been identified in Nicotiana attenuata and Nicotiana tabacum, respectively $[117,118]$. RNA silencing of these genes revealed that COI1 plays an important role in nicotine production. Three JAZ genes have also been identified in N. tabacum, and the direct interaction of NtJAZ1 with NtMYC2 and $\mathrm{NbbHLH} 1 / \mathrm{NbbHLH} 2$ has been confirmed $[26,28,118]$. Hence, it can be concluded that nicotine biosynthesis is regulated by the COI1-JAZ-MYC2 regulatory complex in the JA signaling cascade. Recently, a COI1 homolog has also been reported to be important for the regulation of SGA biosynthesis via GAME9/JRE4 in tomato [119].

The C. roseus COI1 homolog, CrCOI1, has also been reported to interact with several CrJAZ proteins [36]. RNA silencing of the CrCOI1 gene resulted in significant repression of the MeJA-induced upregulation of ORCA3, CrMYC2, BIS1, BIS2, and geraniol 10-hydroxylase $(\mathrm{G} 10 \mathrm{H})$ genes. In contrast, overexpression of $\mathrm{CrCOI1}$ increased the expression levels of biosynthetic enzymes and TF genes involved in MIA biosynthesis and the accumulation of MIAs. These results suggest that the COI1-JAZ-MYC2 complex might control the 
expression of several TF genes to modulate the metabolic pathway of MIA. On the other hand, the involvement of the COI1-JAZ-MYC2 core complex in BIA biosynthesis has not been confirmed, while its presence is suggested in JA signaling.

Protein phosphorylation has also been investigated as a mechanism for the regulation of alkaloid biosynthesis. In tobacco, the induction of JA-factor-stimulating MAPKK1 (JAM1) by MeJA coincided with the expression of NtORC1/ERF221. Co-overexpression of JAM1 with NtORC1 / ERF221 or NbbHLH1 synergistically increased the expression of PMT and QPT promoters in response to MeJA $[13,93]$. These findings suggest that the MAPK phosphorylation cascade is involved in the regulation of nicotine biosynthesis through NtORC1/ERF221 and NbbHLH1.

The expression of MIA biosynthesis genes in response to MeJA was hindered by the addition of protein kinase inhibitors, suggesting the involvement of phosphorylation in the regulation of MIA biosynthesis. The MeJA-responsive MPK3 gene has been identified in C. roseus [120]. Overexpression of CrMPK3 increased the accumulation of several MIAs. More recently, MAP kinase kinase kinase (MAPKKK1), MAP kinase kinase (MAPKK), and MPK6 have also been identified in $C$. roseus [6]. The expression of CrMAPKKK1 and CrMAPKK1 was induced by MeJA, and CrMAPKKK1 interacted with CrMPK3 and CrMPK6. The trans-activation activity of ORCA2-6 and CrMYC2 was significantly enhanced by the co-expression of CrMAPKK1 [8]. Overexpression of CrMAPKK1 showed significant increases in the transcript levels of MIA biosynthetic enzyme genes and ZCT1-3 as well as accumulation of tabersonine and catharanthine. These results suggest that the CrMPK cascade might be involved in the regulation of MIA biosynthesis via phosphorylation of CrMYC2 and ORCA TFs (Figure 3).

Tyrosine phosphorylation and protein degradation have been reported to be involved in the regulation of BIA biosynthesis in C. japonica [121]. The tyrosine phosphorylation of the WRKYGQK core domain in CjWRKY1 affected its DNA-binding activity and subcellular localization, subsequently resulting in CjWRKY1 not being able to activate the transcription of BIA biosynthesis genes. Phosphorylated CjWRKY1, which is localized in the cytosol, might be rapidly degraded by proteases. Furthermore, CjWRKY1, which is not phosphorylated, may also be degraded in the nucleus by the $26 \mathrm{~S}$ proteasome. These findings indicate that BIA biosynthesis might be intensively controlled at the post-translational level, with a combination of protein phosphorylation and degradation (Figure 4).

\section{TFs and Alkaloid Engineering}

TFs are powerful tools for improving production yield and quality by controlling the overall expression of metabolic pathway genes. Since the application of TFs in alkaloid biosynthesis is limited due to a lack of information about the regulatory mechanisms involved, many researchers have tried to explore a master regulator of the biosynthetic pathway and elucidate its function and transcriptional network to achieve alkaloid engineering using several TFs.

For example, ectopic expression of CjWRKY1 in cultured California poppy cells increased the accumulation of several BIAs such as sanguinarine, chelirubine, and chelerythrine in the culture medium, in addition to enhancing the total BIA content up to 2- to 5-fold [113]. The modest effect on alkaloid productivity is due to the post-translational modifications of CjWRKY1. The overexpression of TFs, such as ORCA3 and CrBPF-1 in C. roseus hairy roots, increased the expression levels of several MIA biosynthetic genes, whereas the accumulation levels of MIAs were only slightly altered even after MeJA treatment $[38,122]$. The expression of ZCT genes was also upregulated in these hairy roots, suggesting that the metabolic pathway involved might be rigorously fine-tuned by multiple activators and repressors. Thus, the overexpression of single and native TF genes might be limited to a modest effect on the flux of the biosynthetic pathway. A combinatorial induction of activator genes such as ORCA and $C r M Y C 2$, with the suppression of repressor genes such as ZCT1-3, would be useful in enhancing the metabolic flow of biosynthetic pathways more efficiently. 
Interestingly, Apuya et al. demonstrated the heterologous expression of AtWRKY1 in California poppy and opium poppy cells. Transgenic California poppy cells showed a marked increase in the accumulation of dihydrosanguinarine and 10-hydroxydihydrosanguinarine by up to 30-fold and 34-fold, respectively. Furthermore, the overexpression of AtWRKY1 in opium poppy increased the thebaine content by up to five-fold [115]. These findings suggest that the regulatory TF network in alkaloid biosynthesis might be similar among plant species, but the possible post-translational modifications might be different. Exchange of ERF family TFs between nicotine and MIA pathways has also been reported [7].

Metabolic engineering targeting multiple biosynthetic enzymes and TF genes has also been reported. Wang et al. performed overexpression of ORCA3 in combination with that of $\mathrm{G} 10 \mathrm{H}$, which is not controlled by ORCA3, in C. roseus hairy roots [123]. Transgenic roots showed increased accumulation of catharanthine and expression levels of STR and secologanin synthase (SLS). Co-overexpression of ORCA3 and SGD in C. roseus hairy roots also increased the accumulation levels of several MIAs, such as serpentine and tabersonine, as well as the transcript levels of MIA biosynthetic enzymes and TF genes [124].

The transient overexpression of multiple TF genes, as examined by Schweizer et al. in C. roseus flower petals using agroinfiltration, would be a promising approach to evaluate the modified TF functions in the regulation of biosynthesis. The mutation of CrMYC2 at D126 affects its interaction with JAZ proteins, and CrMYC2 ${ }^{\mathrm{D} 126 \mathrm{~N}}$ strongly induces the expression of genes involved in MIA biosynthesis and transport. Furthermore, combinatorial overexpression of CrMYC2 ${ }^{D 126 N}$, BIS1, and ORCA3 synergistically increased the expression of iridoid and MIA pathway genes and the accumulation levels of several MIAs, including possibly root-specific hörhammericine and 16-methoxyhörhammericine [125].

The above engineering has been mainly performed in de-differentiated cultured cells. However, some alkaloids such as vindoline and morphine are produced by the collaboration of several different cells, such as leaf or root tissues, including epidermal cells, parenchyma cells, and laticifer/idioblast cells. Modification of cell differentiation and in vitro organ culture, especially that of hairy root, might be a more attractive approach to promote metabolic expression since in vitro culture does not require entire plant development. The control of environmental conditions, including light, temperature, and substrate supply, including artificially modified chemicals, could be optimized in vitro with genetic modification. The relationship between tissue/cell-specific expression of biosynthetic enzyme genes and JA-responsive TFs involved in alkaloid biosynthesis is not well studied; however, the detailed tissue/cell-specific regulation of alkaloid biosynthesis may contribute to a novel engineering approach for targeting cell differentiation. In Artemisia annua, an AP2/ERF TF, trichome and artemisinin regulator 1 (TAR1) was reported to regulate the development of trichomes and the biosynthesis of artemisinin, which is produced and stored in trichomes [126]. Recent advances in genetic tools might enable us to manipulate the proliferation of cells involved in the production and accumulation of specific metabolites in the future.

\section{Conclusions}

TFs are central regulators that regulate the expression of genes involved in plant developmental processes and biotic and/or abiotic stress responses. Progress in molecular biological techniques and recent advances in next-generation sequencing technologies have enabled us to perform a comprehensive analysis of TF genes and to explore regulators of alkaloid biosynthesis in several plant species. Indeed, different types of TFs, such as AP2/ERF, WRKY, and bHLH, have been identified in the biosynthesis of each alkaloid and regulatory network, including the MAPK cascade; in addition, post-translational modifications of TFs have also been identified. Some of them are widely distributed in the model plants and are involved in the regulation of secondary metabolism or other plant processes, for example, the MYC2-type bHLH TFs function with COI1 and JAZ proteins in the JA signaling cascade and modulate the expression of genes involved in alkaloid, terpenoid, and anthocyanin biosynthesis. Others are specifically found in alkaloid- 
producing plants, such as non-MYC2-type bHLH TFs in BIA biosynthesis. A comparison of TFs involved in alkaloid biosynthesis with those in terpenoid and phenylpropanoid indicates that the regulatory mechanisms shared in many plants and unique TFs might be evolved in a coordinated manner to form a regulatory network for each biosynthetic pathway. The genome information of many plant species provides some insights into the evolution of transcriptional regulatory networks. AP2/ERF gene clusters have been found in the genomes of C. roseus, N. tabacum, S. lycopersicum, S. tuberosum, and E. californica, but their functions might have differentiated during evolution [18,48]. The evolutionary and biological significance of clustered TF genes is not fully understood; however, biosynthetic genes encoding several classes of enzyme proteins involved in plant-specialized metabolism are often clustered, and their regulation might be involved in the duplicated TFs. An understanding of the regulatory mechanisms associated with clustered TFs in alkaloid biosynthesis, in comparison to those of phenylpropanoid and terpenoid biosynthesis, should be helpful for elucidating the evolution of plant-specialized metabolism and manipulating the specific metabolic pathways resulting in the efficient production of specific metabolites. Furthermore, combinatorial genetic manipulation, such as genome editing and protein modification based on the structure and post-translational regulatory mechanisms, may contribute to a full understanding of the complicated regulatory mechanisms and the development of systems for the efficient production of many valuable metabolites in plants. An elucidation of tissue/cell-specific regulation of genes involved in alkaloid biosynthesis also has the potential to manipulate specific metabolic pathways. Further characterization of TFs that might regulate the expression of genes involved in the compartmentation of valuable metabolites and cell differentiation will be required.

Author Contributions: Conceptualization, Y.Y. and F.S.; investigation, Y.Y. and F.S.; writing—original draft preparation, Y.Y.; writing—review and editing, Y.Y. and F.S.; funding acquisition, Y.Y. and F.S. All authors have read and agreed to the published version of the manuscript.

Funding: This work was supported by the Ministry of Education, Culture, Sports, Science, and Technology of Japan (MEXT) (Grant-in-Aid for Scientific Research (S) 26221201 to F.S. and Grant-inAid for Young Scientists 21K14830 to Y.Y.).

Institutional Review Board Statement: Not applicable.

Informed Consent Statement: Not applicable.

Data Availability Statement: No datasets were generated during the current work.

Acknowledgments: We are thankful to Nobukazu Shitan for his support.

Conflicts of Interest: The authors declare no competing interests.

\section{References}

1. Sato, F. 2.26-Plant Alkaloid Engineering. In Comprehensive Natural Products III; Ben Liu, H.-W., Begley, T.P., Eds.; Elsevier: Oxford, UK, 2020; pp. 700-755. ISBN 9780081026915.

2. Yamada, Y.; Sato, F. Transcription Factors in Alkaloid Biosynthesis. Int. Rev. Cell Mol. Biol. 2013, 305, 339-382.

3. Zhou, M.; Memelink, J. Jasmonate-Responsive Transcription Factors Regulating Plant Secondary Metabolism. Biotechnol. Adv. 2016, 34, 441-449. [CrossRef] [PubMed]

4. Menke, F.L.; Champion, A.; Kijne, J.W.; Memelink, J. A Novel Jasmonate- and Elicitor-Responsive Element in the Periwinkle Secondary Metabolite Biosynthetic Gene Str Interacts with a Jasmonate- and Elicitor-Inducible AP2-Domain Transcription Factor, ORCA2. EMBO J. 1999, 18, 4455-4463. [CrossRef] [PubMed]

5. Van der Fits, L.; Memelink, J. ORCA3, a Jasmonate-Responsive Transcriptional Regulator of Plant Primary and Secondary Metabolism. Science 2000, 289, 295-297. [CrossRef]

6. Paul, P.; Singh, S.K.; Patra, B.; Sui, X.; Pattanaik, S.; Yuan, L. A Differentially Regulated AP2/ERF Transcription Factor Gene Cluster Acts Downstream of a MAP Kinase Cascade to Modulate Terpenoid Indole Alkaloid Biosynthesis in Catharanthus Roseus. New Phytol. 2017, 213, 1107-1123. [CrossRef] [PubMed]

7. Paul, P.; Singh, S.K.; Patra, B.; Liu, X.; Pattanaik, S.; Yuan, L. Mutually Regulated AP2/ERF Gene Clusters Modulate Biosynthesis of Specialized Metabolites in Plants. Plant Physiol. 2020, 182, 840-856. [CrossRef]

8. Singh, S.K.; Patra, B.; Paul, P.; Liu, Y.; Pattanaik, S.; Yuan, L. Revisiting the ORCA Gene Cluster That Regulates Terpenoid Indole Alkaloid Biosynthesis in Catharanthus Roseus. Plant Sci. 2020, 293, 110408. [CrossRef] [PubMed] 
9. Pan, Q.; Wang, C.; Xiong, Z.; Wang, H.; Fu, X.; Shen, Q.; Peng, B.; Ma, Y.; Sun, X.; Tang, K. CrERF5, an AP2/ERF Transcription Factor, Positively Regulates the Biosynthesis of Bisindole Alkaloids and Their Precursors in Catharanthus Roseus. Front. Plant Sci. 2019, 10, 931. [CrossRef] [PubMed]

10. Liu, J.; Gao, F.; Ren, J.; Lu, X.; Ren, G.; Wang, R. A Novel AP2/ERF Transcription Factor CR1 Regulates the Accumulation of Vindoline and Serpentine in Catharanthus Roseus. Front. Plant Sci. 2017, 8, 2082. [CrossRef] [PubMed]

11. Shoji, T.; Kajikawa, M.; Hashimoto, T. Clustered Transcription Factor Genes Regulate Nicotine Biosynthesis in Tobacco. Plant Cell 2010, 22, 3390-3409. [CrossRef]

12. De Sutter, V.; Vanderhaeghen, R.; Tilleman, S.; Lammertyn, F.; Vanhoutte, I.; Karimi, M.; Inzé, D.; Goossens, A.; Hilson, P. Exploration of Jasmonate Signalling via Automated and Standardized Transient Expression Assays in Tobacco Cells. Plant J. 2005, 44, 1065-1076. [CrossRef]

13. De Boer, K.; Tilleman, S.; Pauwels, L.; Vanden Bossche, R.; De Sutter, V.; Vanderhaeghen, R.; Hilson, P.; Hamill, J.D.; Goossens, A. APETALA2/ETHYLENE RESPONSE FACTOR and Basic Helix-Loop-Helix Tobacco Transcription Factors Cooperatively Mediate Jasmonate-Elicited Nicotine Biosynthesis. Plant J. 2011, 66, 1053-1065. [CrossRef] [PubMed]

14. Sears, M.T.; Zhang, H.; Rushton, P.J.; Wu, M.; Han, S.; Spano, A.J.; Timko, M.P. NtERF32: A Non-NIC2 Locus AP2/ERF Transcription Factor Required in Jasmonate-Inducible Nicotine Biosynthesis in Tobacco. Plant Mol. Biol. 2014, 84, 49-66. [CrossRef] [PubMed]

15. Cárdenas, P.D.; Sonawane, P.D.; Pollier, J.; Vanden Bossche, R.; Dewangan, V.; Weithorn, E.; Tal, L.; Meir, S.; Rogachev, I.; Malitsky, S.; et al. GAME9 Regulates the Biosynthesis of Steroidal Alkaloids and Upstream Isoprenoids in the Plant Mevalonate Pathway. Nat. Commun. 2016, 7, 10654. [CrossRef]

16. Thagun, C.; Imanishi, S.; Kudo, T.; Nakabayashi, R.; Ohyama, K.; Mori, T.; Kawamoto, K.; Nakamura, Y.; Katayama, M.; Nonaka, S.; et al. Jasmonate-Responsive ERF Transcription Factors Regulate Steroidal Glycoalkaloid Biosynthesis in Tomato. Plant Cell Physiol. 2016, 57, 961-975. [CrossRef] [PubMed]

17. Udomsom, N.; Rai, A.; Suzuki, H.; Okuyama, J.; Imai, R.; Mori, T.; Nakabayashi, R.; Saito, K.; Yamazaki, M. Function of AP2/ERF Transcription Factors Involved in the Regulation of Specialized Metabolism in Ophiorrhiza Pumila Revealed by Transcriptomics and Metabolomics. Front. Plant Sci. 2016, 7, 1861. [CrossRef] [PubMed]

18. Yamada, Y.; Nishida, S.; Shitan, N.; Sato, F. Genome-Wide Identification of AP2/ERF Transcription Factor-Encoding Genes in California Poppy (Eschscholzia Californica) and Their Expression Profiles in Response to Methyl Jasmonate. Sci. Rep. 2020, 10, 18066. [CrossRef]

19. Kato, N.; Dubouzet, E.; Kokabu, Y.; Yoshida, S.; Taniguchi, Y.; Dubouzet, J.G.; Yazaki, K.; Sato, F. Identification of a WRKY Protein as a Transcriptional Regulator of Benzylisoquinoline Alkaloid Biosynthesis in Coptis Japonica. Plant Cell Physiol. 2007, 48, 8-18. [CrossRef]

20. Suttipanta, N.; Pattanaik, S.; Kulshrestha, M.; Patra, B.; Singh, S.K.; Yuan, L. The Transcription Factor CrWRKY1 Positively Regulates the Terpenoid Indole Alkaloid Biosynthesis in Catharanthus Roseus. Plant Physiol. 2011, 157, 2081-2093. [CrossRef]

21. Mishra, S.; Triptahi, V.; Singh, S.; Phukan, U.J.; Gupta, M.M.; Shanker, K.; Shukla, R.K. Wound Induced Tanscriptional Regulation of Benzylisoquinoline Pathway and Characterization of Wound Inducible PsWRKY Transcription Factor from Papaver Somniferum. PLOS ONE 2013, 8, e52784.

22. Xu, M.; Wu, C.; Zhao, L.; Wang, Y.; Wang, C.; Zhou, W.; Ming, Y.; Kai, G. WRKY Transcription Factor OpWRKY1 Acts as a Negative Regulator of Camptothecin Biosynthesis in Ophiorrhiza Pumila Hairy Roots. Plant Cell Tissue Organ Cult. 2020, 142, 69-78. [CrossRef]

23. Hao, X.; Xie, C.; Ruan, Q.; Zhang, X.; Wu, C.; Han, B.; Qian, J.; Zhou, W.; Nützmann, H.-W.; Kai, G. The Transcription Factor OpWRKY2 Positively Regulates the Biosynthesis of the Anticancer Drug Camptothecin in Ophiorrhiza Pumila. Hortic. Res. 2021, 8, 7. [CrossRef] [PubMed]

24. Wang, C.; Wu, C.; Wang, Y.; Xie, C.; Shi, M.; Nile, S.; Zhou, Z.; Kai, G. Transcription Factor OpWRKY3 Is Involved in the Development and Biosynthesis of Camptothecin and Its Precursors in Ophiorrhiza Pumila Hairy Roots. Int. J. Mol. Sci. 2019, 20, 3996. [CrossRef]

25. Todd, A.T.; Liu, E.; Polvi, S.L.; Pammett, R.T.; Page, J.E. A Functional Genomics Screen Identifies Diverse Transcription Factors That Regulate Alkaloid Biosynthesis in Nicotiana Benthamiana. Plant J. 2010, 62, 589-600. [CrossRef]

26. Zhang, H.-B.; Bokowiec, M.T.; Rushton, P.J.; Han, S.-C.; Timko, M.P. Tobacco Transcription Factors NtMYC2a and NtMYC2b Form Nuclear Complexes with the NtJAZ1 Repressor and Regulate Multiple Jasmonate-Inducible Steps in Nicotine Biosynthesis. Mol. Plant 2012, 5, 73-84. [CrossRef] [PubMed]

27. Sui, X.; He, X.; Song, Z.; Gao, Y.; Zhao, L.; Jiao, F. The Gene NtMYC2a Acts as a "Master Switch" in the Regulation of JA-induced Nicotine Accumulation in Tobacco. Plant 2021, 23, 317-326. [CrossRef] [PubMed]

28. Shoji, T.; Hashimoto, T. Tobacco MYC2 Regulates Jasmonate-Inducible Nicotine Biosynthesis Genes Directly and by Way of the NIC2-Locus ERF Genes. Plant Cell Physiol. 2011, 52, 1117-1130. [CrossRef]

29. Chatel, G.; Montiel, G.; Pré, M.; Memelink, J.; Thiersault, M.; Saint-Pierre, B.; Doireau, P.; Gantet, P. CrMYC1, a Catharanthus Roseus Elicitor-and Jasmonate-Responsive bHLH Transcription Factor That Binds the G-Box Element of the Strictosidine Synthase Gene Promoter. J. Exp. Bot. 2003, 54, 2587-2588. [CrossRef] 
30. Zhang, H.; Hedhili, S.; Montiel, G.; Zhang, Y.; Chatel, G.; Pré, M.; Gantet, P.; Memelink, J. The Basic Helix-Loop-Helix Transcription Factor CrMYC2 Controls the Jasmonate-Responsive Expression of the ORCA Genes That Regulate Alkaloid Biosynthesis in Catharanthus Roseus. Plant J. 2011, 67, 61-71. [CrossRef]

31. Van Moerkercke, A.; Steensma, P.; Schweizer, F.; Pollier, J.; Gariboldi, I.; Payne, R.; Vanden Bossche, R.; Miettinen, K.; Espoz, J.; Purnama, P.C.; et al. The bHLH Transcription Factor BIS1 Controls the Iridoid Branch of the Monoterpenoid Indole Alkaloid Pathway in Catharanthus Roseus. Proc. Natl. Acad. Sci. USA 2015, 112, 8130-8135. [CrossRef] [PubMed]

32. Van Moerkercke, A.; Steensma, P.; Gariboldi, I.; Espoz, J.; Purnama, P.C.; Schweizer, F.; Miettinen, K.; Vanden Bossche, R.; De Clercq, R.; Memelink, J.; et al. The Basic Helix-Loop-Helix Transcription Factor BIS2 Is Essential for Monoterpenoid Indole Alkaloid Production in the Medicinal Plant Catharanthus Roseus. Plant J. 2016, 88, 3-12. [CrossRef]

33. Singh, S.K.; Patra, B.; Paul, P.; Liu, Y.; Pattanaik, S.; Yuan, L. BHLH IRIDOID SYNTHESIS 3 Is a Member of a bHLH Gene Cluster Regulating Terpenoid Indole Alkaloid Biosynthesis in Catharanthus Roseus. Plant Direct 2021, 5, e00305. [CrossRef] [PubMed]

34. Yamada, Y.; Kokabu, Y.; Chaki, K.; Yoshimoto, T.; Ohgaki, M.; Yoshida, S.; Kato, N.; Koyama, T.; Sato, F. Isoquinoline Alkaloid Biosynthesis Is Regulated by a Unique bHLH-Type Transcription Factor in Coptis Japonica. Plant Cell Physiol. 2011, 52, 1131-1141. [CrossRef]

35. Yamada, Y.; Motomura, Y.; Sato, F. CjbHLH1 Homologs Regulate Sanguinarine Biosynthesis in Eschscholzia Californica Cells. Plant Cell Physiol. 2015, 56, 1019-1030. [CrossRef] [PubMed]

36. Patra, B.; Pattanaik, S.; Schluttenhofer, C.; Yuan, L. A Network of Jasmonate-Responsive bHLH Factors Modulate Monoterpenoid Indole Alkaloid Biosynthesis in Catharanthus Roseus. New Phytol. 2018, 217, 1566-1581. [CrossRef]

37. Van der Fits, L.; Zhang, H.; Menke, F.L.H.; Deneka, M.; Memelink, J. A Catharanthus Roseus BPF-1 Homologue Interacts with an Elicitor-Responsive Region of the Secondary Metabolite Biosynthetic Gene Str and Is Induced by Elicitor via a JA-Independent Signal Transduction Pathway. Plant Mol. Biol. 2000, 44, 675-685. [CrossRef] [PubMed]

38. Li, C.Y.; Leopold, A.L.; Sander, G.W.; Shanks, J.V.; Zhao, L.; Gibson, S.I. CrBPF1 Overexpression Alters Transcript Levels of Terpenoid Indole Alkaloid Biosynthetic and Regulatory Genes. Front. Plant Sci. 2015, 6, 818. [CrossRef]

39. Rohani, E.R.; Chiba, M.; Kawaharada, M.; Asano, T.; Oshima, Y.; Mitsuda, N.; Ohme-Takagi, M.; Fukushima, A.; Rai, A.; Saito, K.; et al. An MYB Transcription Factor Regulating Specialized Metabolisms in Ophiorrhiza Pumila. Plant Biotechnol. 2016, 33, 1-9. [CrossRef]

40. Sibéril, Y.; Benhamron, S.; Memelink, J.; Giglioli-Guivarc'h, N.; Thiersault, M.; Boisson, B.; Doireau, P.; Gantet, P. Catharanthus Roseus G-Box Binding Factors 1 and 2 Act as Repressors of Strictosidine Synthase Gene Expression in Cell Cultures. Plant Mol. Biol. 2001, 45, 477-488. [CrossRef]

41. Pauw, B.; Hilliou, F.A.O.; Martin, V.S.; Chatel, G.; de Wolf, C.J.F.; Champion, A.; Pré, M.; van Duijn, B.; Kijne, J.W.; van der Fits, L.; et al. Zinc Finger Proteins Act as Transcriptional Repressors of Alkaloid Biosynthesis Genes in Catharanthus Roseus. J. Biol. Chem. 2004, 279, 52940-52948. [CrossRef]

42. Liu, Y.; Patra, B.; Pattanaik, S.; Wang, Y.; Yuan, L. GATA and Phytochrome Interacting Factor Transcription Factors Regulate Light-Induced Vindoline Biosynthesis in Catharanthus Roseus. Plant Physiol. 2019, 180, 1336-1350. [CrossRef]

43. Vom Endt, D.; Soares e Silva, M.; Kijne, J.W.; Pasquali, G.; Memelink, J. Identification of a Bipartite Jasmonate-Responsive Promoter Element in the Catharanthus Roseus ORCA3 Transcription Factor Gene That Interacts Specifically with AT-Hook DNA-Binding Proteins. Plant Physiol. 2007, 144, 1680-1689. [CrossRef] [PubMed]

44. Nakano, T.; Suzuki, K.; Fujimura, T.; Shinshi, H. Genome-Wide Analysis of the ERF Gene Family in Arabidopsis and Rice. Plant Physiol. 2006, 140, 411-432. [CrossRef] [PubMed]

45. Mizoi, J.; Shinozaki, K.; Yamaguchi-Shinozaki, K. AP2/ERF Family Transcription Factors in Plant Abiotic Stress Responses. Biochim. Biophys. Acta 2012, 1819, 86-96. [CrossRef] [PubMed]

46. Lu, X.; Tang, K.; Li, P. Plant Metabolic Engineering Strategies for the Production of Pharmaceutical Terpenoids. Front. Plant Sci. 2016, 7, 1647. [CrossRef]

47. Zhang, Y.; Ji, A.; Xu, Z.; Luo, H.; Song, J. The AP2/ERF Transcription Factor SmERF128 Positively Regulates Diterpenoid Biosynthesis in Salvia Miltiorrhiza. Plant Mol. Biol. 2019, 100, 83-93. [CrossRef]

48. Shoji, T.; Yuan, L. ERF Gene Clusters: Working Together to Regulate Metabolism. Trends Plant Sci. 2021, 26, 23-32. [CrossRef] [PubMed]

49. Eulgem, T.; Somssich, I.E. Networks of WRKY Transcription Factors in Defense Signaling. Curr. Opin. Plant Biol. 2007, 10, 366-371. [CrossRef] [PubMed]

50. Rushton, P.J.; Somssich, I.E.; Ringler, P.; Shen, Q.J. WRKY Transcription Factors. Trends Plant Sci. 2010, 15, 247-258. [CrossRef]

51. Eulgem, T.; Rushton, P.J.; Robatzek, S.; Somssich, I.E. The WRKY Superfamily of Plant Transcription Factors. Trends Plant Sci. 2000, 5, 199-206. [CrossRef]

52. Yamasaki, K.; Kigawa, T.; Watanabe, S.; Inoue, M.; Yamasaki, T.; Seki, M.; Shinozaki, K.; Yokoyama, S. Structural Basis for Sequence-Specific DNA Recognition by an Arabidopsis WRKY Transcription Factor. J. Biol. Chem. 2012, 287, 7683-7691. [CrossRef] [PubMed]

53. Yamasaki, K.; Kigawa, T.; Seki, M.; Shinozaki, K.; Yokoyama, S. DNA-Binding Domains of Plant-Specific Transcription Factors: Structure, Function, and Evolution. Trends Plant Sci. 2013, 18, 267-276. [CrossRef] [PubMed] 
54. Ma, D.; Pu, G.; Lei, C.; Ma, L.; Wang, H.; Guo, Y.; Chen, J.; Du, Z.; Wang, H.; Li, G.; et al. Isolation and Characterization of AaWRKY1, an Artemisia Annua Transcription Factor That Regulates the Amorpha-4, 11-Diene Synthase Gene, a Key Gene of Artemisinin Biosynthesis. Plant Cell Physiol. 2009, 50, 2146-2161. [CrossRef] [PubMed]

55. Atchley, W.R.; Fitch, W.M. A Natural Classification of the Basic Helix-loop-helix Class of Transcription Factors. Proc. Natl. Acad. Sci. USA 1997, 94, 5172-5176. [CrossRef] [PubMed]

56. Stevens, J.D.; Roalson, E.H.; Skinner, M.K. Phylogenetic and Expression Analysis of the Basic Helix-Loop-Helix Transcription Factor Gene Family: Genomic Approach to Cellular Differentiation. Differentiation 2008, 76, 1006-1022. [CrossRef]

57. Heim, M.A.; Jakoby, M.; Werber, M.; Martin, C.; Weisshaar, B.; Bailey, P.C. The Basic Helix-Loop-Helix Transcription Factor Family in Plants: A Genome-Wide Study of Protein Structure and Functional Diversity. Mol. Biol. Evol. 2003, $20,735-747$. [CrossRef]

58. Carretero-Paulet, L.; Galstyan, A.; Roig-Villanova, I.; Martínez-García, J.F.; Bilbao-Castro, J.R.; Robertson, D.L. Genome-Wide Classification and Evolutionary Analysis of the bHLH Family of Transcription Factors in Arabidopsis, Poplar, Rice, Moss, and Algae. Plant Physiol. 2010, 153, 1398-1412. [CrossRef]

59. Buck, M.J.; Atchley, W.R. Phylogenetic Analysis of Plant Basic Helix-Loop-Helix Proteins. J. Mol. Evol. 2003, 56, 742-750. [CrossRef]

60. Ludwig, S.R.; Habera, L.F. Lc, a Member of the Maize R Gene Family Responsible for Tissue-Specific Anthocyanin Production, Encodes a Protein Similar to Transcriptional Activators and Contains the Myc-Homology Region. Proc. Natl. Acad. Sci. USA 1989, 86, 7092-7096. [CrossRef]

61. Koes, R.; Verweij, W.; Quattrocchio, F. Flavonoids: A Colorful Model for the Regulation and Evolution of Biochemical Pathways. Trends Plant Sci. 2005, 10, 236-242. [CrossRef]

62. Goossens, J.; Mertens, J.; Goossens, A. Role and Functioning of bHLH Transcription Factors in Jasmonate Signalling. J. Exp. Bot. 2017, 68, 1333-1347. [CrossRef]

63. Lorenzo, O.; Chico, J.M.; Sánchez-Serrano, J.J.; Solano, R. JASMONATE-INSENSITIVE1 Encodes a MYC Transcription Factor Essential to Discriminate between Different Jasmonate-Regulated Defense Responses in Arabidopsis. Plant Cell 2004, 16, 1938-1950. [CrossRef]

64. Dombrecht, B.; Xue, G.P.; Sprague, S.J.; Kirkegaard, J.A.; Ross, J.J.; Reid, J.B.; Fitt, G.P.; Sewelam, N.; Schenk, P.M.; Manners, J.M.; et al. MYC2 Differentially Modulates Diverse Jasmonate-Dependent Functions in Arabidopsis. Plant Cell 2007, 19, $2225-2245$. [CrossRef]

65. Chini, A.; Fonseca, S.; Fernández, G.; Adie, B.; Chico, J.M.; Lorenzo, O.; García-Casado, G.; López-Vidriero, I.; Lozano, F.M.; Ponce, M.R.; et al. The JAZ Family of Repressors Is the Missing Link in Jasmonate Signalling. Nature 2007, 448, 666-671. [CrossRef] [PubMed]

66. Thines, B.; Katsir, L.; Melotto, M.; Niu, Y.; Mandaokar, A.; Liu, G.; Nomura, K.; He, S.Y.; Howe, G.A.; Browse, J. JAZ Repressor Proteins Are Targets of the SCF(COI1) Complex during Jasmonate Signalling. Nature 2007, 448, 661-665. [CrossRef] [PubMed]

67. Zhou, Y.; Sun, W.; Chen, J.; Tan, H.; Xiao, Y.; Li, Q.; Ji, Q.; Gao, S.; Chen, L.; Chen, S.; et al. SmMYC2a and SmMYC2b Played Similar but Irreplaceable Roles in Regulating the Biosynthesis of Tanshinones and Phenolic Acids in Salvia Miltiorrhiza. Sci. Rep. 2016, 6, 22852. [CrossRef] [PubMed]

68. Shen, Q.; Lu, X.; Yan, T.; Fu, X.; Lv, Z.; Zhang, F.; Pan, Q.; Wang, G.; Sun, X.; Tang, K. The Jasmonate-Responsive AaMYC2 Transcription Factor Positively Regulates Artemisinin Biosynthesis in Artemisia Annua. New Phytol. 2016, 210, $1269-1281$. [CrossRef] [PubMed]

69. Yamada, Y.; Koyama, T.; Sato, F. Basic Helix-Loop-Helix Transcription Factors and Regulation of Alkaloid Biosynthesis. Plant Signal. Behav. 2011, 6, 1627-1630. [CrossRef]

70. Mertens, J.; Pollier, J.; Vanden Bossche, R.; Lopez-Vidriero, I.; Franco-Zorrilla, J.M.; Goossens, A. The bHLH Transcription Factors TSAR1 and TSAR2 Regulate Triterpene Saponin Biosynthesis in Medicago Truncatula. Plant Physiol. 2016, 170, 194-210. [CrossRef] [PubMed]

71. Tamura, K.; Yoshida, K.; Hiraoka, Y.; Sakaguchi, D.; Chikugo, A.; Mochida, K.; Kojoma, M.; Mitsuda, N.; Saito, K.; Muranaka, T.; et al. The Basic Helix-Loop-Helix Transcription Factor GubHLH3 Positively Regulates Soyasaponin Biosynthetic Genes in Glycyrrhiza Uralensis. Plant Cell Physiol. 2018, 59, 783-796. [CrossRef]

72. Frerigmann, H.; Glawischnig, E.; Gigolashvili, T. The Role of MYB34, MYB51 and MYB122 in the Regulation of Camalexin Biosynthesis in Arabidopsis Thaliana. Front. Plant Sci. 2015, 6, 654. [CrossRef] [PubMed]

73. Xu, W.; Dubos, C.; Lepiniec, L. Transcriptional Control of Flavonoid Biosynthesis by MYB-bHLH-WDR Complexes. Trends Plant Sci. 2015, 20, 176-185. [CrossRef] [PubMed]

74. Grotewold, E. The Genetics and Biochemistry of Floral Pigments. Annu. Rev. Plant Biol. 2006, 57, 761-780. [CrossRef] [PubMed]

75. Teng, S.; Keurentjes, J.; Bentsink, L.; Koornneef, M.; Smeekens, S. Sucrose-Specific Induction of Anthocyanin Biosynthesis in Arabidopsis Requires the MYB75/PAP1 Gene. Plant Physiol. 2005, 139, 1840-1852. [CrossRef]

76. Loreti, E.; Povero, G.; Novi, G.; Solfanelli, C.; Alpi, A.; Perata, P. Gibberellins, Jasmonate and Abscisic Acid Modulate the Sucrose-Induced Expression of Anthocyanin Biosynthetic Genes in Arabidopsis. New Phytol. 2008, 179, 1004-1016. [CrossRef] [PubMed]

77. Xie, Y.; Tan, H.; Ma, Z.; Huang, J. DELLA Proteins Promote Anthocyanin Biosynthesis via Sequestering MYBL2 and JAZ Suppressors of the MYB/bHLH/WD40 Complex in Arabidopsis Thaliana. Mol. Plant 2016, 9, 711-721. [CrossRef] [PubMed] 
78. Shan, X.; Zhang, Y.; Peng, W.; Wang, Z.; Xie, D. Molecular Mechanism for Jasmonate-Induction of Anthocyanin Accumulation in Arabidopsis. J. Exp. Bot. 2009, 60, 3849-3860. [CrossRef] [PubMed]

79. Qi, T.; Song, S.; Ren, Q.; Wu, D.; Huang, H.; Chen, Y.; Fan, M.; Peng, W.; Ren, C.; Xie, D. The Jasmonate-ZIM-Domain Proteins Interact with the WD-Repeat/bHLH/MYB Complexes to Regulate Jasmonate-Mediated Anthocyanin Accumulation and Trichome Initiation in Arabidopsis Thaliana. Plant Cell 2011, 23, 1795-1814. [CrossRef] [PubMed]

80. Jeong, S.-W.; Das, P.K.; Jeoung, S.C.; Song, J.-Y.; Lee, H.K.; Kim, Y.-K.; Kim, W.J.; Park, Y.I.; Yoo, S.-D.; Choi, S.-B.; et al. Ethylene Suppression of Sugar-Induced Anthocyanin Pigmentation in Arabidopsis. Plant Physiol. 2010, 154, 1514-1531. [CrossRef]

81. Meng, L.-S.; Xu, M.-K.; Wan, W.; Yu, F.; Li, C.; Wang, J.-Y.; Wei, Z.-Q.; Lv, M.-J.; Cao, X.-Y.; Li, Z.-Y.; et al. Sucrose Signaling Regulates Anthocyanin Biosynthesis Through a MAPK Cascade in Arabidopsis Thaliana. Genetics 2018, 210, 607-619. [CrossRef]

82. Brown, B.A.; Cloix, C.; Jiang, G.H.; Kaiserli, E.; Herzyk, P.; Kliebenstein, D.J.; Jenkins, G.I. A UV-B-Specific Signaling Component Orchestrates Plant UV Protection. Proc. Natl. Acad. Sci. USA 2005, 102, 18225-18230. [CrossRef]

83. Huang, X.; Ouyang, X.; Yang, P.; Lau, O.S. Conversion from CUL4-Based COP1-SPA E3 Apparatus to UVR8-COP1-SPA Complexes Underlies a Distinct Biochemical Function of COP1 under UV-B. Proc. Natl. Acad. Sci. USA 2013, 110, 16669-16674. [CrossRef] [PubMed]

84. Shin, J.; Park, E.; Choi, G. PIF3 Regulates Anthocyanin Biosynthesis in an HY5-Dependent Manner with Both Factors Directly Binding Anthocyanin Biosynthetic Gene Promoters in Arabidopsis. Plant J. 2007, 49, 981-994. [CrossRef]

85. Shin, D.H.; Choi, M.; Kim, K.; Bang, G.; Cho, M.; Choi, S.-B.; Choi, G.; Park, Y.-I. HY5 Regulates Anthocyanin Biosynthesis by Inducing the Transcriptional Activation of the MYB75/PAP1 Transcription Factor in Arabidopsis. FEBS Lett. 2013, 587, 1543-1547. [CrossRef]

86. Pichersky, E.; Gershenzon, J. The Formation and Function of Plant Volatiles: Perfumes for Pollinator Attraction and Defense. Curr. Opin. Plant Biol. 2002, 5, 237-243. [CrossRef]

87. Hong, G.-J.; Xue, X.-Y.; Mao, Y.-B.; Wang, L.-J.; Chen, X.-Y. Arabidopsis MYC2 Interacts with DELLA Proteins in Regulating Sesquiterpene Synthase Gene Expression. Plant Cell 2012, 24, 2635-2648. [CrossRef] [PubMed]

88. Hansen, B.G.; Halkier, B.A. New Insight into the Biosynthesis and Regulation of Indole Compounds in Arabidopsis Thaliana. Planta 2005, 221, 603-606. [CrossRef] [PubMed]

89. Mao, G.; Meng, X.; Liu, Y.; Zheng, Z.; Chen, Z.; Zhang, S. Phosphorylation of a WRKY Transcription Factor by Two PathogenResponsive MAPKs Drives Phytoalexin Biosynthesis in Arabidopsis. Plant Cell 2011, 23, 1639-1653. [CrossRef] [PubMed]

90. Saga, H.; Ogawa, T.; Kai, K.; Suzuki, H.; Ogata, Y.; Sakurai, N.; Shibata, D.; Ohta, D. Identification and Characterization of ANAC042, a Transcription Factor Family Gene Involved in the Regulation of Camalexin Biosynthesis in Arabidopsis. Mol. Plant. Microbe Interact. 2012, 25, 684-696. [CrossRef] [PubMed]

91. Schuhegger, R.; Rauhut, T.; Glawischnig, E. Regulatory Variability of Camalexin Biosynthesis. J. Plant Physiol. 2007, 164, 636-644. [CrossRef]

92. Baccelli, I.; Lombardi, L.; Luti, S.; Bernardi, R.; Picciarelli, P.; Scala, A.; Pazzagli, L. Cerato-Platanin Induces Resistance in Arabidopsis Leaves through Stomatal Perception, Overexpression of Salicylic Acid- and Ethylene-Signalling Genes and Camalexin Biosynthesis. PLoS ONE 2014, 9, e100959. [CrossRef] [PubMed]

93. Goossens, A.; Häkkinen, S.T.; Laakso, I.; Seppänen-Laakso, T.; Biondi, S.; De Sutter, V.; Lammertyn, F.; Nuutila, A.M.; Söderlund, H.; Zabeau, M.; et al. A Functional Genomics Approach toward the Understanding of Secondary Metabolism in Plant Cells. Proc. Natl. Acad. Sci. USA 2003, 100, 8595-8600. [CrossRef]

94. Rushton, P.J.; Bokowiec, M.T.; Han, S.; Zhang, H.; Brannock, J.F.; Chen, X.; Laudeman, T.W.; Timko, M.P. Tobacco Transcription Factors: Novel Insights into Transcriptional Regulation in the Solanaceae. Plant Physiol. 2008, 147, 280-295. [CrossRef] [PubMed]

95. Hayashi, S.; Watanabe, M.; Kobayashi, M.; Tohge, T.; Hashimoto, T.; Shoji, T. Genetic Manipulation of Transcriptional Regulators Alters Nicotine Biosynthesis in Tobacco. Plant Cell Physiol. 2020, 61, 1041-1053. [CrossRef] [PubMed]

96. Shoji, T.; Hashimoto, T. DNA-Binding and Transcriptional Activation Properties of Tobacco NIC2-Locus ERF189 and Related Transcription Factors. Plant Biotechnol. 2012, 29, 35-42. [CrossRef]

97. Shoji, T.; Hashimoto, T. Recruitment of a Duplicated Primary Metabolism Gene into the Nicotine Biosynthesis Regulon in Tobacco. Plant J. 2011, 67, 949-959. [CrossRef] [PubMed]

98. Nakayasu, M.; Shioya, N.; Shikata, M.; Thagun, C.; Abdelkareem, A.; Okabe, Y.; Ariizumi, T.; Arimura, G.-I.; Mizutani, M.; Ezura, H.; et al. JRE4 Is a Master Transcriptional Regulator of Defense-Related Steroidal Glycoalkaloids in Tomato. Plant J. 2018, 94, 975-990. [CrossRef]

99. Li, C.Y.; Leopold, A.L.; Sander, G.W.; Shanks, J.V.; Zhao, L.; Gibson, S.I. The ORCA2 Transcription Factor Plays a Key Role in Regulation of the Terpenoid Indole Alkaloid Pathway. BMC Plant Biol. 2013, 13, 155. [CrossRef] [PubMed]

100. Van Der Fits, L.; Memelink, J. The Jasmonate-Inducible AP2/ERF-Domain Transcription Factor ORCA3 Activates Gene Expression via Interaction with a Jasmonate-Responsive Promoter Element. Plant J. 2001, 25, 43-53. [PubMed]

101. Sui, X.; Singh, S.K.; Patra, B.; Schluttenhofer, C.; Guo, W.; Pattanaik, S.; Yuan, L. Cross-Family Transcription Factor Interaction between MYC2 and GBFs Modulates Terpenoid Indole Alkaloid Biosynthesis. J. Exp. Bot. 2018, 69, 4267-4281. [CrossRef] [PubMed]

102. Rizvi, N.F.; Weaver, J.D.; Cram, E.J.; Lee-Parsons, C.W.T. Silencing the Transcriptional Repressor, ZCT1, Illustrates the Tight Regulation of Terpenoid Indole Alkaloid Biosynthesis in Catharanthus Roseus Hairy Roots. PLoS ONE 2016, 11, e0159712. [CrossRef] [PubMed] 
103. Chen, Y.-Y.; Wang, L.-F.; Dai, L.-J.; Yang, S.-G.; Tian, W.-M. Characterization of HbEREBP1, a Wound-Responsive Transcription Factor Gene in Laticifers of Hevea Brasiliensis Muell. Arg. Mol. Biol. Rep. 2012, 39, 3713-3719. [CrossRef]

104. Wang, Y.; Zhan, D.-F.; Li, H.-L.; Guo, D.; Zhu, J.-H.; Peng, S.-Q. Transcriptome-Wide Identification and Characterization of MYB Transcription Factor Genes in the Laticifer Cells of Hevea Brasiliensis. Front. Plant Sci. 2017, 8, 1974. [CrossRef] [PubMed]

105. Singh, A.; Menéndez-Perdomo, I.M.; Facchini, P.J. Benzylisoquinoline Alkaloid Biosynthesis in Opium Poppy: An Update. Phytochem. Rev. 2019, 18, 1457-1482. [CrossRef]

106. Guo, L.; Winzer, T.; Yang, X.; Li, Y.; Ning, Z.; He, Z.; Teodor, R.; Lu, Y.; Bowser, T.A.; Graham, I.A.; et al. The Opium Poppy Genome and Morphinan Production. Science 2018, 362, 343-347. [CrossRef]

107. Filiault, D.L.; Ballerini, E.S.; Mandáková, T.; Aköz, G.; Derieg, N.J.; Schmutz, J.; Jenkins, J.; Grimwood, J.; Shu, S.; Hayes, R.D.; et al. The Aquilegia Genome Provides Insight into Adaptive Radiation and Reveals an Extraordinarily Polymorphic Chromosome with a Unique History. Elife 2018, 7, e36426. [CrossRef] [PubMed]

108. Liu, X.; Liu, Y.; Huang, P.; Ma, Y.; Qing, Z.; Tang, Q.; Cao, H.; Cheng, P.; Zheng, Y.; Yuan, Z.; et al. The Genome of Medicinal Plant Macleaya Cordata Provides New Insights into Benzylisoquinoline Alkaloids Metabolism. Mol. Plant 2017, 10, 975-989. [CrossRef] [PubMed]

109. Hori, K.; Yamada, Y.; Purwanto, R.; Minakuchi, Y.; Toyoda, A.; Hirakawa, H.; Sato, F. Mining of the Uncharacterized Cytochrome P450 Genes Involved in Alkaloid Biosynthesis in California Poppy Using a Draft Genome Sequence. Plant Cell Physiol. 2018, 59, 222-233. [CrossRef]

110. Ming, R.; VanBuren, R.; Liu, Y.; Yang, M.; Han, Y.; Li, L.-T.; Zhang, Q.; Kim, M.-J.; Schatz, M.C.; Campbell, M.; et al. Genome of the Long-Living Sacred Lotus (Nelumbo Nucifera Gaertn.). Genome Biol. 2013, 14, R41. [CrossRef]

111. Liu, Y.; Wang, B.; Shu, S.; Li, Z.; Song, C.; Liu, D.; Niu, Y.; Liu, J.; Zhang, J.; Liu, H.; et al. Analysis of the Coptis Chinensis Genome Reveals the Diversification of Protoberberine-Type Alkaloids. Nat. Commun. 2021, 12, 3276. [CrossRef]

112. Yamada, Y.; Hirakawa, H.; Hori, K.; Minakuchi, Y.; Toyoda, A.; Shitan, N.; Sato, F. Comparative Analysis Using the Draft Genome Sequence of California Poppy (Eschscholzia Californica) for Exploring the Candidate Genes Involved in Benzylisoquinoline Alkaloid Biosynthesis. Biosci. Biotechnol. Biochem. 2021, 85, 851-859. [CrossRef] [PubMed]

113. Yamada, Y.; Shimada, T.; Motomura, Y.; Sato, F. Modulation of Benzylisoquinoline Alkaloid Biosynthesis by Heterologous Expression of CjWRKY1 in Eschscholzia Californica Cells. PLoS ONE 2017, 12, e0186953. [CrossRef] [PubMed]

114. Yamada, Y.; Nishida, S.; Shitan, N.; Sato, F. Genome-Wide Profiling of WRKY Genes Involved in Benzylisoquinoline Alkaloid Biosynthesis in California Poppy (Eschscholzia Californica). Front. Plant Sci. 2021, 12. [CrossRef] [PubMed]

115. Apuya, N.R.; Park, J.-H.; Zhang, L.; Ahyow, M.; Davidow, P.; Van Fleet, J.; Rarang, J.C.; Hippley, M.; Johnson, T.W.; Yoo, H.-D.; et al. Enhancement of Alkaloid Production in Opium and California Poppy by Transactivation Using Heterologous Regulatory Factors. Plant Biotechnol. J. 2008, 6, 160-175. [CrossRef] [PubMed]

116. Yamada, Y.; Yoshimoto, T.; Yoshida, S.T.; Sato, F. Characterization of the Promoter Region of Biosynthetic Enzyme Genes Involved in Berberine Biosynthesis in Coptis Japonica. Front. Plant Sci. 2016, 7, 1352. [CrossRef]

117. Paschold, A.; Halitschke, R.; Baldwin, I.T. Co(i)-Ordinating Defenses: NaCOI1 Mediates Herbivore- Induced Resistance in Nicotiana Attenuata and Reveals the Role of Herbivore Movement in Avoiding Defenses. Plant J. 2007, 51, 79-91. [CrossRef] [PubMed]

118. Shoji, T.; Ogawa, T.; Hashimoto, T. Jasmonate-Induced Nicotine Formation in Tobacco Is Mediated by Tobacco COI1 and JAZ Genes. Plant Cell Physiol. 2008, 49, 1003-1012. [CrossRef] [PubMed]

119. Abdelkareem, A.; Thagun, C.; Nakayasu, M.; Mizutani, M.; Hashimoto, T.; Shoji, T. Jasmonate-Induced Biosynthesis of Steroidal Glycoalkaloids Depends on COI1 Proteins in Tomato. Biochem. Biophys. Res. Commun. 2017, 489, 206-210. [CrossRef] [PubMed]

120. Raina, S.K.; Wankhede, D.P.; Jaggi, M.; Singh, P.; Jalmi, S.K.; Raghuram, B.; Sheikh, A.H.; Sinha, A.K. CrMPK3, a Mitogen Activated Protein Kinase from Catharanthus Roseus and Its Possible Role in Stress Induced Biosynthesis of Monoterpenoid Indole Alkaloids. BMC Plant Biol. 2012, 12, 134. [CrossRef] [PubMed]

121. Yamada, Y.; Sato, F. Tyrosine Phosphorylation and Protein Degradation Control the Transcriptional Activity of WRKY Involved in Benzylisoquinoline Alkaloid Biosynthesis. Sci. Rep. 2016, 6, 31988. [CrossRef] [PubMed]

122. Peebles, C.A.M.; Hughes, E.H.; Shanks, J.V.; San, K.-Y. Transcriptional Response of the Terpenoid Indole Alkaloid Pathway to the Overexpression of ORCA3 along with Jasmonic Acid Elicitation of Catharanthus Roseus Hairy Roots over Time. Metab. Eng. 2009, 11, 76-86. [CrossRef] [PubMed]

123. Wang, C.-T.; Liu, H.; Gao, X.-S.; Zhang, H.-X. Overexpression of G10H and ORCA3 in the Hairy Roots of Catharanthus Roseus Improves Catharanthine Production. Plant Cell Rep. 2010, 29, 887-894. [CrossRef] [PubMed]

124. Sun, J.; Peebles, C.A.M. Engineering Overexpression of ORCA3 and Strictosidine Glucosidase in Catharanthus Roseus Hairy Roots Increases Alkaloid Production. Protoplasma 2016, 253, 1255-1264. [CrossRef] [PubMed]

125. Schweizer, F.; Colinas, M.; Pollier, J.; Van Moerkercke, A.; Vanden Bossche, R.; de Clercq, R.; Goossens, A. An Engineered Combinatorial Module of Transcription Factors Boosts Production of Monoterpenoid Indole Alkaloids in Catharanthus Roseus. Metab. Eng. 2018, 48, 150-162. [CrossRef] [PubMed]

126. Tan, H.; Xiao, L.; Gao, S.; Li, Q.; Chen, J.; Xiao, Y.; Ji, Q.; Chen, R.; Chen, W.; Zhang, L. TRICHOME AND ARTEMISININ REGULATOR 1 Is Required for Trichome Development and Artemisinin Biosynthesis in Artemisia Annua. Mol. Plant 2015, 8 , 1396-1411. [CrossRef] [PubMed] 